\title{
A Jordan-Brouwer Separation Theorem for Polyhedral Pseudomanifolds
}

\author{
Micha A. Perles · Horst Martini • Yaakov S. Kupitz
}

Received: 6 June 2008 / Revised: 24 February 2009 / Accepted: 8 April 2009 /

Published online: 16 May 2009

(C) Springer Science+Business Media, LLC 2009

\begin{abstract}
The Jordan Curve Theorem referring to a simple closed curve in the plane has a particularly simple proof in the case that the curve is polygonal, called the "raindrop proof". We generalize the notion of a simple closed polygon to that of a polyhedral $(d-1)$-pseudomanifold $(d \geq 2)$ and prove a Jordan-Brouwer Separation Theorem for such a manifold embedded in $\mathbb{R}^{d}$. As a by-product, we get bounds on the polygonal diameter of the interior and exterior of such a manifold which are almost tight. This puts the result within the frame of computational geometry.
\end{abstract}

Keywords Bing's house - Dual graph · Euler's formula $\cdot$ Jordan-Brouwer theorem · Jordan's curve theorem · Jordan exterior (interior) · Polygonal diameter $\cdot$ Polyhedral manifold $\cdot$ Triangulation

\section{Introduction}

Jordan's Curve Theorem asserts that if $\gamma$ is a simple closed curve in $\mathbb{R}^{2}$, then $\mathbb{R}^{2} \backslash \gamma$ is the disjoint union of two non-empty connected open sets ("domains" in the old terminology), and that $\gamma$ is the common boundary of these two domains. Moreover, the bounded component of $\mathbb{R}^{2} \backslash \gamma$ is simply connected (this follows from the JordanSchönflies Theorem, see [32] or [23, p. 68]), and a one-point compactification of $\mathbb{R}^{2}$

The research of Y.S. Kupitz was partially supported by the Landau Center at the Mathematics Institute of the Hebrew University of Jerusalem (supported by Minerva Foundation, Germany), and by Deutsche Forschungsgemeinschaft.

M.A. Perles · Y.S. Kupitz

Institute of Mathematics, The Hebrew University of Jerusalem, Jerusalem, Israel

H. Martini ( $\square)$

Faculty of Mathematics, University of Technology, 09107 Chemnitz, Germany

e-mail: horst.martini@mathematik.tu-chemnitz.de 
transforming it into an $S^{2}$ results in a one-point extension of the unbounded component of $\mathbb{R}^{2} \backslash \gamma$, which again is simply connected, see [23, p. 71]. The separation property of $\gamma$ assured by the Jordan Curve Theorem does generalize to $(d-1)$-spheres in $\mathbb{R}^{d}, d \geq 3$ (or in $S^{d}$ ) (Jordan-Brouwer Separation Theorem, see [31, p. 198, Theorem 15]), but for $d \geq 3$ the two domains separated by $S^{d-1}$ embedded in $\mathbb{R}^{d}$ (or $S^{d}$ ) are not necessarily simply connected ("Alexander's horned sphere", first described in [2]). For various proofs of Jordan's basic (and deep) Curve Theorem, we refer to [8, 20, 32], [23, p. $31 \mathrm{ff}$, [3, Vol. I, pp. 39-64], [19, pp. $285 \mathrm{ff}]$, and the survey [11]. In the case when $\gamma$ is a simple closed polygon (in the plane), this theorem specializes to the Jordan theorem for simple closed polygons which has a particularly simple proof, known as the "raindrop proof". (See [10, pp. 267-269], [15, pp. 281-285], [7, pp. 27-29], or [23, pp. 16-18]. An interesting historical article is [14].) The purpose of this paper is to extend the theorem and its "raindrop proof" (for the case of a simple closed polygon) to $d$-dimensional space $\mathbb{R}^{d}$. In Definition 1.3 below, we shall define a polyhedral $(d-1)$-pseudomanifold (abbreviated $(d-1)-P P M)$ in $\mathbb{R}^{d}$, and we shall prove, for these pseudomanifolds, the following

Theorem 1.1 (Jordan-Brouwer Separation Theorem for polyhedral $(d-1)$-pseudomanifolds) If $\mathcal{C}$ is a $(d-1)-P P M$ in $\mathbb{R}^{d}(d \geq 1)$ and $|\mathcal{C}|$ denotes the body of $\mathcal{C}$ (= the union of all cells of $\mathcal{C}$ ), then $\mathbb{R}^{d} \backslash|\mathcal{C}|$ is the disjoint union of two non-empty connected open sets (= "domains"), one bounded and one unbounded, and $|\mathcal{C}|$ is the common boundary of these two components.

For related results in $\mathbb{R}^{3}$ see [9] and [17], and for the Jordan-Brouwer Theorem in $\mathbb{R}^{d}, d \geq 2$, see [12].

Definition 1.1 The bounded component of $\mathbb{R}^{d} \backslash|\mathcal{C}|$ is the interior, denoted by int $\mathcal{C}$ of $\mathcal{C}$, and the unbounded component, denoted by $\operatorname{ext} \mathcal{C}$, is the exterior of $\mathcal{C}$.

Historical notes Theorem 1.1 is a special case of the Jordan-Brouwer Separation Theorem for $(d-1)$-pseudomanifolds in $\mathbb{R}^{d}$ formulated in the mid 1940s, perhaps earlier, and proved by homology methods (see below). The main novelty of Theorem 1.1 over the general Jordan-Brouwer Theorem is its pure polyhedral formulation and its elementary "raindrop" proof, which makes it accessible to every geometer who does not feel at home with the machinery of algebraic topology. The relative easiness and transparency of this proof over the complications that arise in the general case may be well illustrated by the planar case, where the "raindrop" proof is much easier than the proof of the Jordan theorem for general curves. Moreover, this raindrop proof serves to compute the maximal polygonal diameter of the interior (resp., exterior) of a $(d-1)-P P M$, which puts it in the frame of computational geometry not accessible by the methods of algebraic topology. The question of the maximal polygonal diameter of $\operatorname{int} \mathcal{C}$ (resp., ext $\mathcal{C}$ ) for a general $(d-1)$-pseudomanifold $\mathcal{C}$ in $\mathbb{R}^{d}$ is, in fact, meaningless (it is unbounded even for $C^{\infty}$-smooth manifolds).

For the sake of historical accuracy, we give now one of the earliest formulations of the Jordan-Brouwer Separation Theorem for $(d-1)$-pseudomanifolds which we could find in the literature (quote): 
The Jordan-Brouwer Theorem "Every $(d-1)$-dimensional closed pseudomanifold in $S^{d}$ is orientable (and) separates $S^{d}$ into precisely two domains ("domain" is the old terminology for "open connected set") and is the common boundary of these two domains" (see [3, Vol. III, p. 54, Theorem 3.44]). (The proof uses homology theory.)

In fact, our "polyhedral $(d-1)$-pseudomanifold" $(=(d-1)-P P M)$ is essentially the piecewise linear (PL) structure underlying Aleksandrov's " $d$-dimensional pseudomanifold" defined in ibid, Vol. II, p. 72, Definition 3.11. Aleksandrov's "strongly connected $d$-complex" there is essentially the condition on the connectedness of the dual graph (our strong connectivity condition (D) below; see ibid, Vol. I, p. 200, Definition 5.24), and what makes it there into a "pseudomanifold" is the condition that every subfacet is common to exactly two facets, i.e., our no branching condition (C) below. (In ibid, p. 72, Definition 3.12 "exactly" is replaced by "either one or two" (hence, not zero), a generalization which makes the pseudomanifold into a "pseudomanifold with boundary".) Since Aleksandrov's book [3] is relatively old, we tried to find out whether the Jordan-Brouwer Separation Theorem for $(d-1)$-pseudomanifolds appears in more recent books, and we could not find anything. For example, [31, p. 198, Theorem 15] has simply a $(d-1)$-sphere embedded in $S^{d}$, whereas [24, p. 446, Corollary 74.2] has a "compact connected triangulable $(d-1)$-manifold" (without singularities) embedded in $S^{d}$, and on p. 425, Corollary 71.2, it has a homeomorph $A$ of $S^{d-1}$ embedded in $S^{d}$ such that " $\left(S^{d}, A\right)$ is triangulable", which is a certain condition needed for the variant of the homology theory developed there.

The reader may justifiably ask now (as indeed one of our referees did) whether this "raindrop proof" is not merely a specialization of the homology proof to our piecewise linear setting. The answer is definitely "no". The "raindrop proof" does not mimic any part of the homology approach. It uses a sort of height function, thus resembling a Morse's theory approach rather than a homology approach, where the $(d-1)$-manifold is gradually sliced by parallel hyperplanes so that the singularities encountered in this process are scrutinized. Such a process was used already in [1] to show that a 2-manifold of genus 1 in $\mathbb{R}^{3}$ separates $\mathbb{R}^{3}$ into two components one of which is homeomorphic to the interior of a 2-torus ("tubular region" in Alexander's terminology) as conjectured by H. Tietze. Alexander's proof is, however, very sketchy.

Definition 1.2 A polyhedral cell complex $\mathcal{C}$ in $\mathbb{R}^{d}$ is a finite non-empty collection of convex polytopes (= cells) in $\mathbb{R}^{d}$ that satisfies the following two conditions:

(A) If $P \in \mathcal{C}$ and if $F$ is a face of $P$ (i.e., the intersection of $P$ with a supporting hyperplane), then $F \in \mathcal{C}$ as well.

(B) If $P, Q \in \mathcal{C}$, then the intersection $P \cap Q$ is a face of both $P$ and $Q$.

If $P \in \mathcal{C}$ has dimension $k$, then we refer to $P$ as a $k$-cell of $\mathcal{C}$. The dimension of $\mathcal{C}$ is defined as $\max \{\operatorname{dim} P: P \in \mathcal{C}\}$, and $|\mathcal{C}|$, the body of $\mathcal{C}$, is defined as the union of all cells of $\mathcal{C}$. A cell $Q \in \mathcal{C}$ is maximal if $Q$ is not included in any other cell of $\mathcal{C}$. The cell complex $\mathcal{C}$ is homogeneously $k$-dimensional if all maximal cells of $\mathcal{C}$ are $k$-dimensional. The dual graph of a homogeneously $k$-dimensional complex $\mathcal{C}$ is defined as follows: its vertices are the $k$-cells of $\mathcal{C}$. Two distinct vertices are joined by an edge if the corresponding $k$-cells share a $(k-1)$-face. 
Fig. 1 A 2- $P P M$ embedded in $\mathbb{R}^{3}$

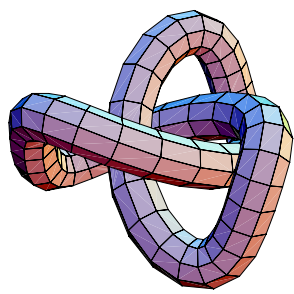

Definition 1.3 A $(d-1)$-dimensional polyhedral pseudomanifold (abbr. $(d-1)$ $P P M)$ in $\mathbb{R}^{d}$ is a homogeneously $(d-1)$-dimensional polyhedral complex in $\mathbb{R}^{d}$ that satisfies the following conditions:

(C) (No branching) Every $(d-2)$-cell is included in exactly two $(d-1)$-cells.

(D) (Strong connectivity) The dual graph is connected.

If $\mathcal{C}$ is a $(d-1)-P P M$ in $\mathbb{R}^{d}$, then the $(d-1)$-cells of $\mathcal{C}$ are the facets and the $(d-2)$ cells are the subfacets of $\mathcal{C}$. We denote by vert $\mathcal{C}$ the set of vertices of $\mathcal{C}$, by $\operatorname{skel}_{k}(\mathcal{C})$ the $k$-skeleton of $\mathcal{C}(0 \leq k \leq d)$, and by $f_{k}(\mathcal{C})$ the number of $k$-cells of $\mathcal{C}$.

\section{Remark 1.1}

(1) Notice that we do not assume any regularity conditions on the cells of dimension $d-3$ or less. Thus, for $d \geq 3$ the body $|\mathcal{C}|$ of a $(d-1)-P P M \mathcal{C}$ may be homeomorphic to a pseudomanifold, such as, e.g., a "degenerate torus" consisting of a $(d-1)$-sphere with two diametrical points identified. An easy realization of this $(d-1)$-pseudomanifold as the body $|\mathcal{C}|$ of a $(d-1)-P P M$ is the following: Let $P$ be a $d$-prism with (parallel) bases $Q, Q^{\prime}$ and barycenter $c$. The complex $\mathcal{C}$ is obtained from the boundary complex $\mathcal{B}(P)$ of $P$ by removing the facets $Q, Q^{\prime}$ and adding $\{c\}$ and all pyramids $\operatorname{conv}(\{c\} \cup F)$ (with apex $c$ and base $F$ ), where $F$ ranges over all proper faces of $Q$ and of $Q^{\prime}$.

(2) For $d=3$, the body of a 2-PPM $\mathcal{C}$ (even without singularities) may be homeomorphic to an arbitrary closed orientable 2-manifold, i.e., a 2-sphere with a finite number of handles. Another example of a $2-P P M$ is the trefoil knot $(=3$-leaf clover) in the logo of the Mathematics Faculty of the Chemnitz University of Technology; see Fig. 1. In [6], it is proved that the Heawood map of seven countries on the torus is not realizable as a 2-PPM in $\mathbb{R}^{3}$, and in [29] it is proved that the dual $(3,7)_{8}$ of the Klein map $(7,3)_{8}$ is realizable as a $2-P P M$ in $\mathbb{R}^{3}$. The authors note that the Klein map $(7,3)_{8}$ itself is not realizable in such a way; see 1.c., p. 541, lines 27-31. In fact, as follows from [13, Exercise 13.2.3 on p. 253], no simple 2-map of genus $g \geq 1$ (such as the Klein map $\{7,3\}_{8}$ ) is realizable in any $\mathbb{R}^{d}$ (when we require the faces to be convex).

(3) Theorem 1.1 trivially holds for $d=1$. For $d=2$, a $(d-1)-P P M$ is just a simple closed polygon, and the theorem reduces to Jordan's theorem for simple closed polygons in the plane.

(4) For $d \geq 3$, a polyhedral $(d-1)$-complex $\mathcal{C}$ in $\mathbb{R}^{d}$ whose dual graph is connected (i.e., $\mathcal{C}$ satisfies condition (D) above) and in which every $(d-1)$-cell is included in at least two $(d-1)$-cells (instead of exactly two $(d-1)$-cells as in condition 


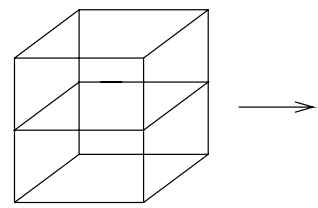

a

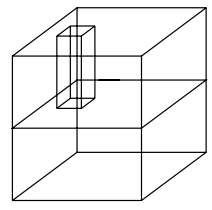

$\mathrm{b}$

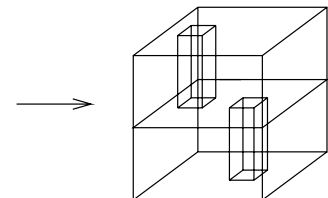

$\mathrm{C}$

Fig. 2 Simplified Bing's house

(C) above), the complement $\mathbb{R}^{d} \backslash \mathcal{C}$ may be connected, as the following example shows.

Example 1.1 (Simplified Bing's house with two rooms) Begin with the boundary of a 3-cube, to which we add in the middle a horizontal (2-dimensional square) barrier (Fig. 2(a)). So far this is a polyhedral 2-dimensional complex which separates $\mathbb{R}^{3}$ into three components (the exterior and two floors inside).

Next make a square hole near the "north-west" corner of the upper ceiling, just below it we make a parallel hole in the middle ceiling, and between these two holes form a "chimney" in the upper floor by adding four vertical panels as shown in Fig. 2(b).

A person standing on the roof may fall now into the lower storey through the chimney. Repeat the construction symmetrically near the opposite "south-east" corner in the lower floor. The polyhedral 2-complex $\mathcal{C}$ obtained (see Fig. 2(c)) has a connected dual graph, every edge of $\mathcal{C}$ is contained in at least two facets, and $\mathcal{C}$ does not separate $\mathbb{R}^{3}$, i.e., $\mathbb{R}^{3} \backslash|\mathcal{C}|$ is connected. This example easily generalizes to $d \geq 3$.

(5) It is well known that if $\gamma$ is a simple closed polygon in $\mathbb{R}^{2}$, then $\gamma \cup$ int $\gamma$ can be triangulated without adding vertices (see, e.g., [15], pp. 286-287). This does not generalize to $(d-1)$-PPM's in $\mathbb{R}^{d}$ for $d \geq 3$, i.e., there is a $2-P P M C$ in $\mathbb{R}^{3}$ such that $\mathcal{C} \cup$ int $\mathcal{C}$ cannot be tetrahedrized without additional vertices. Two such 2-PPM's in $\mathbb{R}^{3}$ are presented in Sect. 5 below. But other results based on the piecewise Jordan separation theorem may be generalized using Theorem 1.1, e.g., the characterization of those geometric graphs which are 1-skeletons of unstacked triangulations of simple closed polygons given in [18].

For the sake of the proof of Theorem 1.1, we split our main theorem into two statements, namely: Let $\mathcal{C}$ be a $(d-1)-P P M$ in $\mathbb{R}^{d}$. Then

(E) $\mathbb{R}^{d} \backslash|\mathcal{C}|$ is the disjoint union of two open sets, int $\mathcal{C}$ and $\operatorname{ext} \mathcal{C}$. The boundary of each of these sets is $|\mathcal{C}|$; int $\mathcal{C}$ is bounded, and $\operatorname{ext} \mathcal{C}$ is unbounded.

(F) The sets int $\mathcal{C}$ and $\operatorname{ext} \mathcal{C}$ are (polygonally) connected.

We shall prove (E) in Sect. 2 by constructing a continuous function $f: \mathbb{R}^{d} \backslash|\mathcal{C}| \rightarrow$ $\{0,1\}$ which attains both values 0 and 1 in every neighborhood of every points $x \in|\mathcal{C}|$, and defining $\operatorname{ext} \mathcal{C}=f^{-1}(0)$, int $\mathcal{C}=f^{-1}(1)$. The (polygonal) connectedness of int $\mathcal{C}$ and $\operatorname{ext} \mathcal{C}$ is proved in Sect. 3 (see in particular Theorem 3.2).

Notation $B^{k}(c, r)$ denotes the closed ball of dimension $k$ centered at $c$ and having radius $r$. 


\section{A "Raindrop" Proof of (E)}

The construction of $f$ will be performed in four steps.

Step I: Choosing a "generic" direction.

Let $F_{1}, \ldots, F_{t}$ be all hyperplanes $(=(d-1)$-flats) spanned by subsets of vert $\mathcal{C}$. For $i=1, \ldots, t$, let $F_{i}^{0}={ }_{\text {def }} F_{i}-F_{i}$ be the linear subspace parallel to $F_{i}$. Choose a unit vector $v \in \mathbb{R}^{d} \backslash \bigcup_{i=1}^{t} F_{i}^{0}$ (" $v$ " for "vertical"). Vector $v$ is our direction "up", and $-v$ is pointing "down".

By the choice of $v$, a flat $F$ spanned by vertices of $\mathcal{C}$, other than $\mathbb{R}^{d}$, will meet a line parallel to $v$ in at most one point. For each point $p \in \mathbb{R}^{d} \backslash|\mathcal{C}|$, denote by $R(p)$ the closed vertical "pointing down" half-line $R(p)=_{\text {def }}\{p-\lambda v: 0 \leq \lambda<\infty\} . R(p)$ is the path of a "raindrop" emanating from $p$. We divide $\mathbb{R}^{d} \backslash|\mathcal{C}|$ into three disjoint sets (recall that a subfacet means a $(d-2)$-cell):

$$
\begin{aligned}
& S_{0}=\left\{p \in \mathbb{R}^{d} \backslash|\mathcal{C}|: R(p) \text { does not meet any subfacet of } \mathcal{C}\right\}, \\
& S_{1}=\left\{p \in \mathbb{R}^{d} \backslash|\mathcal{C}|: R(p) \text { meets exactly one subfacet of } \mathcal{C}\right\}, \\
& S_{2}=\left\{p \in \mathbb{R}^{d} \backslash|\mathcal{C}|: R(p) \text { meets more than one subfacet of } \mathcal{C}\right\} .
\end{aligned}
$$

We shall first define $f$ on $S_{0}$ (= Step II), then extend its definition (continuously) to $S_{1}$ (= Step III), and finally extend it (continuously) to $S_{2}$ (= Step IV).

In the sequel, we shall use the following notation: For a set $A \subset \mathbb{R}^{d}, A^{+}=\mathrm{def}_{\mathrm{de}}$ $\{a+\lambda v: a \in A, \lambda \geq 0\}$. $A^{+}$is the set of points of $\mathbb{R}^{d}$ that lie "above" $A$. Note that (for all $p \in \mathbb{R}^{d}$ and $A \subset \mathbb{R}^{d}$ ):

$$
R(p) \text { meets } A \text { iff } p \in A^{+} .
$$

Step II: Define $f$ on $S_{0}$.

For $p \in S_{0}$, denote by $r(p)$ the number of facets $(=(d-1)$-cells of $\mathcal{C})$ that meet $R(p)$, and define $f(p)={ }_{\text {def }} \frac{1}{2}\left(1-(-1)^{r(p)}\right)$ to be the parity of $r(p)(f(p)=0$ if $r(p)$ is even, 1 if $r(p)$ is odd).

Next we show that $S_{0}$ is a dense open subset of $\mathbb{R}^{d}$, and that $f: S_{0} \rightarrow\{0,1\}$ is a continuous, hence locally constant, function. In view of (1), we can write $S_{0}=$ $\mathbb{R}^{d} \backslash\left(|\mathcal{C}| \cup\left|\operatorname{skel}_{d-2} \mathcal{C}\right|^{+}\right)$, where $\left|\operatorname{skel}_{d-2} \mathcal{C}\right|$ denotes the union of all subfacets of $\mathcal{C}$. The set $\left|\operatorname{skel}_{d-2} \mathcal{C}\right|$ is compact, same as $\mathcal{C}$, and therefore $\left|\operatorname{skel}_{d-2} \mathcal{C}\right|^{+}$is closed. Thus $S_{0}$ is an open subset of $\mathbb{R}^{d}$. Moreover, the set $|\mathcal{C}| \cup \mid$ skel $\left._{d-2} \mathcal{C}\right|^{+}$can be covered by a finite number of hyperplanes in $\mathbb{R}^{d}$. It follows that $S_{0}$ is dense in $\mathbb{R}^{d}$.

Now for the continuity of $f$. Assume $x \in S_{0}$. Let $\varepsilon$ be the (positive) distance from $x$ to $|\mathcal{C}| \cup\left|\operatorname{skel}_{d-2} \mathcal{C}\right|^{+}\left(=\mathbb{R}^{d} \backslash S_{0}\right)$. If $x^{\prime} \in \mathbb{R}^{d},\left\|x-x^{\prime}\right\|<\varepsilon$, then the segment $\left[x, x^{\prime}\right]$ does not meet $|\mathcal{C}| \cup\left|\operatorname{skel}_{d-2} \mathcal{C}\right|^{+}$. Let $F$ be any facet of $\mathcal{C}$. The set $F^{+}$is a closed, convex, unbounded and full-dimensional polyhedral subset of $\mathbb{R}^{d}$ whose boundary consists of the base $F$ and of the side facets $G^{+}$, where $G$ ranges over all $(d-2)$ faces of $F$. Thus, bd $F^{+} \subset|\mathcal{C}| \cup\left|\operatorname{skel}_{d-2} \mathcal{C}\right|^{+}$, and therefore the segment $\left[x, x^{\prime}\right]$ does not meet the boundary of $F^{+}$. It follows that $x^{\prime} \in F^{+}$iff $x \in F^{+}$, i.e., $R(x)$ meets $F$ iff $R\left(x^{\prime}\right)$ meets $F$. This is true for all facets $F$ of $\mathcal{C}$. Therefore, $r(x)=r\left(x^{\prime}\right)$, 
hence $f\left(x^{\prime}\right)=f(x)$. Thus we have shown that the function $f: S_{0} \rightarrow\{0,1\}$ is locally constant, hence continuous.

Step III: Extend f continuously from $S_{0}$ to $S_{0} \cup S_{1}$.

Suppose $p \in S_{1}$. Let $G$ be the unique subfacet of $\mathcal{C}$ that meets $R(p)$ (or, equivalently, such that $\left.p \in G^{+}\right)$. $R(p)$ meets $G$ in a unique point $g \in \operatorname{relint} G$. (The uniqueness of $g$ follows from our choice of the vertical direction. If $g$ were not a relative interior point of $G$, then $g$ would belong to a proper face $J$ of $G$, which, in turn, would be included in another subfacet $G^{\prime}$, contradicting our assumption that $R(p)$ meets only one subfacet.) Hence $p \in \operatorname{relint} G^{+}$.

Let $F_{1}, F_{2}$ be the facets of $\mathcal{C}$ that include $G$. Define $H=$ def aff $G+\mathbb{R} v . H$ is the vertical hyperplane through $G$. Denote by $H^{-}, H^{+}$the two closed half-spaces of $\mathbb{R}^{d}$ bounded by $H$. The two facets $F_{1}, F_{2}$ may be in the same half-space, or in different half-spaces. Choose the notation in such a way that either $(\alpha) F_{1} \subset H^{-}, F_{2} \subset H^{+}$ or $(\beta) F_{1} \cup F_{2} \subset H^{+}$.

Now consider the closed set $\Delta==_{\text {def }}|\mathcal{C}| \cup \mid \operatorname{skel}_{d-2}(\mathcal{C}) \backslash$ relint $\left.G\right|^{+}$. (Here $\mid \operatorname{skel}_{d-2}(\mathcal{C}) \backslash$ relint $G \mid$ is the union of all subfacets of $\mathcal{C}$, except relint $G$.) This set includes the boundary of $F^{+}$, for every facet $F$ of $\mathcal{C}$, except for $F_{1}^{+}$and $F_{2}^{+}$. It also includes the boundaries of $F_{1}^{+}$and $F_{2}^{+}$, except for $\operatorname{relint}\left(G^{+}\right)$. In particular, it includes the relative boundary of $G^{+}$. But it does not contain the point $p$. Put $\varepsilon={ }_{\operatorname{def}} \operatorname{dist}(p, \Delta)$, and define $U={ }_{\operatorname{def}}\left\{x \in \mathbb{R}^{d}:\|x-p\|<\varepsilon\right\}=$ int $B^{d}(p, \varepsilon)$. Note that if $x \in U$, then the closed interval $[p, x]$ does not meet $\Delta$. Now make the following observations.

(I) If $F$ is any facet of $\mathcal{C}$, other than $F_{1}$ and $F_{2}$, then the interval $[p, x]$ does not meet the boundary of $F^{+}$, and therefore $p$ and $x$ are either both in $F^{+}$, or both not in $F^{+}$.

(II) If, say, $F_{1} \subset H^{-}$and $x \in$ int $H^{-}$then, moving along the interval $[p, x]$ from $p$ to $x$, we start at a point $p \in \operatorname{relint} G^{+} \subset$ bd $F_{1}^{+}$, move into int $F_{1}^{+}$, and do not hit the boundary of $F_{1}^{+}$again. Therefore, $x \in \operatorname{int} F_{1}^{+}$. The same holds with $H^{-}$ replaced by $H^{+}$, and/or $F_{1}$ replaced by $F_{2}$. It follows in case $(\alpha)$ : if $x \in U \backslash H$, then $x$ belongs to exactly one of the sets $F_{1}^{+}, F_{2}^{+}$. And it follows in case $(\beta)$ : if $x \in U \cap$ int $H^{-}$, then $x$ belongs to none of the sets $F_{1}^{+}, F_{2}^{+}$; if $x \in U \cap H^{+}$, then $x$ belongs to both.

(III) If $G^{\prime}$ is any subfacet of $\mathcal{C}$ other than $G$, then $G^{\prime+} \subset \Delta$, and therefore $x \notin G^{\prime+}$, for any $x \in U$.

(IV) If $x \in U \backslash H$, then clearly $x \notin G^{+}$. If $x \in U \cap H$, then the interval [ $\left.p, x\right]$ lies in aff $G^{+}(=H)$, contains a point $p \in \operatorname{relint} G^{+}$, and does not meet the relative boundary of $G^{+}$(which is part of $\Delta$ ); therefore, $x \in \operatorname{relint} G^{+}$.

From these observations we conclude:

(A) $U \backslash H \subset S_{0}$, and $f$ is constant on $U \backslash H$.

(B) $U \cap H \subset S_{1}$.

Now define $f(p)$ to be the constant value that $f$ takes on $U \backslash H$. Clearly, if we apply the same procedure to any point $q \in U \cap H$, we will end up with a value $f(q)$ equal to the value $f(p)$ just defined. Thus we have extended $f$ to a continuous function $f: S_{0} \cup S_{1} \rightarrow\{0,1\}$. 
Step IV: Extend $f$ continuously from $S_{0} \cup S_{1}$ to $S_{0} \cup S_{1} \cup S_{2}\left(=\mathbb{R}^{d} \backslash|\mathcal{C}|\right)$.

Assume $p \in S_{2}$. Then the ray $R(p)$ meets at least two subfacets of $\mathcal{C}$. (This can happen only for $d \geq 3$, by our choice of $v$.) Assume that $R(p)$ meets exactly $t$ distinct subfacets $G_{1}, \ldots, G_{t}$ of $\mathcal{C}, t \geq 2$. Denote by $\mathcal{G}$ the subcomplex of $\mathcal{C}$ that consists of all cells that do not meet $R(p)$. Then $p \notin|\mathcal{C}| \cup|\mathcal{G}|^{+}$. Define $\varepsilon=\operatorname{def} \operatorname{dist}\left(p,|\mathcal{C}| \cup|\mathcal{G}|^{+}\right)$, and let $U={ }_{\operatorname{def}} B^{d}(p, \varepsilon) \subset \mathbb{R}^{d}$ (the open ball of radius $\varepsilon$ centered at $p$ ). By our choice of $\varepsilon$, we have $S_{2} \cap U=\bigcup_{i=1}^{t} \bigcup_{j=i+1}^{t}\left(G_{i}^{+} \cap G_{j}^{+} \cap U\right)$. In Lemma 2.1 below, we will show that each of the sets $G_{i}^{+} \cap G_{j}^{+}(1 \leq i<j \leq t)$ is included in a $(d-2)$ flat. This will imply (see Lemma 2.2 below) that the union of these sets does not separate $U$. The integer valued function $f$, defined and continuous on the connected set $\left(S_{0} \cup S_{1}\right) \cap U\left(=U \backslash \bigcup_{i=1}^{t} \bigcup_{j=i+1}^{t}\left(G_{i}^{+} \cap G_{j}^{+}\right)\right.$, takes a constant value $c(c=0$ or $c=1)$ there. We define $f(p)$ to be $c$ as well. This rule, applied to any other point $q \in S_{2} \cap U$, will assign to $q$ the same value $f(q)=c$. Thus we have extended $f$ to a continuous (hence locally constant) function from $S_{0} \cup S_{1} \cup S_{2}\left(=\mathbb{R}^{d} \backslash|\mathcal{C}|\right)$ to $\{0,1\}$.

To complete the proof of statement $(\mathrm{E})$, we define, as indicated above, the sets $\operatorname{ext} \mathcal{C}=\operatorname{def} f^{-1}(0)$ and $\operatorname{int} \mathcal{C}=\operatorname{def} f^{-1}(1)$. These are clearly two disjoint open sets in $\mathbb{R}^{d}$, whose union is $\operatorname{dom} f=\mathbb{R}^{d} \backslash|\mathcal{C}|$. Note that $\mathbb{R}^{d} \backslash \operatorname{conv}|\mathcal{C}| \subset \operatorname{ext} \mathcal{C}$, and therefore $\operatorname{int} \mathcal{C} \subset \operatorname{conv}|\mathcal{C}|$. Thus ext $\mathcal{C}$ is unbounded, and $\operatorname{int} \mathcal{C}$ is bounded.

We still have to show that every point of $|\mathcal{C}|$ is a common boundary point of int $\mathcal{C}$ and $\operatorname{ext} \mathcal{C}$ (and therefore int $\mathcal{C} \neq \emptyset$, ext $\mathcal{C} \neq \emptyset$ ). Since the boundaries of int $\mathcal{C}$ and of $\operatorname{ext} \mathcal{C}$ are closed sets, it suffices to show that the common boundary points of int $\mathcal{C}$ and $\operatorname{ext} \mathcal{C}$ are dense in $|\mathcal{C}|$. If $G$ is a subfacet $(=(d-2)$-face) of $\mathcal{C}$, then the set $G+\mathbb{R} v$ is included in a vertical hyperplane, and therefore intersects a facet $F$ of $\mathcal{C}$ in a convex set of dimension $\leq d-2$. Thus $F \backslash \cup\left\{G+\mathbb{R} v: G \in \operatorname{skel}_{d-2} \mathcal{C}\right\}$ is dense in $F$, and $|\mathcal{C}| \backslash\left(\mathbb{R} v+\left|\operatorname{skel}_{d-2} \mathcal{C}\right|\right)$ is dense in $|\mathcal{C}|$. If $x \in|\mathcal{C}| \backslash\left(\mathbb{R} v+\left|\operatorname{skel}_{d-2} \mathcal{C}\right|\right)$, then $x$ belongs to the relative interior of some facet $F$ of $\mathcal{C}$. If $\varepsilon>0$ is sufficiently small, then the points $x+\varepsilon v, x-\varepsilon v$ are both in $S_{0}$, the ray $R(x+\varepsilon v)$ meets $F$, in addition to all facets met by $R(x-\varepsilon v)$. Thus $r(x+\varepsilon v)=1+r(x-\varepsilon v)$, and $f(x+\varepsilon v) \neq f(x-\varepsilon v)$, i.e., $\{f(x-\varepsilon v), f(x+\varepsilon v)\}=\{0,1\}$. Thus $x$ is a common boundary point of int $\mathcal{C}$ and $\operatorname{ext} \mathcal{C}$.

Lemma 2.1 For $1 \leq i<j \leq t$ we have $\operatorname{dim}\left(G_{i}^{+} \cap G_{j}^{+}\right) \leq d-2$.

Proof Case I. If $G_{i}^{+} \cap G_{j}^{+}=\left(G_{i} \cap G_{j}\right)^{+}$, then

$$
\operatorname{dim}\left(G_{i}^{+} \cap G_{j}^{+}\right)=\operatorname{dim}\left(G_{i} \cap G_{j}\right)^{+}=1+\operatorname{dim}\left(G_{i} \cap G_{j}\right) \leq 1+(d-3)=d-2 .
$$

(The intersection of two distinct $(d-2)$-cells of $\mathcal{C}$ is a cell of $\mathcal{C}$, of dimension $\leq$ $d-3$.)

Case II. $G_{i}^{+} \cap G_{j}^{+} \supsetneqq\left(G_{i} \cap G_{j}\right)^{+}$.

This means that we can find a point $q$ that lies above a point $q^{\prime} \in G_{i}$, and above a point $q^{\prime \prime} \in G_{j}, q^{\prime} \neq q^{\prime \prime}$. Thus $q^{\prime}=q-\lambda^{\prime} v, q^{\prime \prime}=q-\lambda^{\prime \prime} v, \lambda^{\prime}>0, \lambda^{\prime \prime}>0$, $\lambda^{\prime} \neq \lambda^{\prime \prime}$. The sets (aff $\left.G_{i}\right)^{+}$and (aff $\left.G_{j}\right)^{+}$are both $(d-1)$ )-flats. If they are distinct, then $G_{i}^{+} \cap G_{j}^{+}$is included in their intersection, which is a $(d-2)$-flat. There remains the possibility that $\left(\operatorname{aff} G_{i}\right)^{+}=\left(\operatorname{aff} G_{j}\right)^{+}={ }_{\text {def }} H$. If this happens, then the set 
$W=$ def vert $G_{i} \cup \operatorname{vert} G_{j}$ is included in the $(d-1)$-flat $H$, and the difference set of aff $W$ contains the vector $q^{\prime}-q^{\prime \prime}=\left(\lambda^{\prime}-\lambda^{\prime \prime}\right) v$, contrary to our choice of $v$.

Lemma 2.2 Let $U \subset \mathbb{R}^{d}$ be a convex set with non-empty interior $(\operatorname{dim} U=d)$. For $i=1, \ldots, t$, let $J_{i}$ be a $(d-2)$-flat in $\mathbb{R}^{d}$, and let $S_{i}$ be a subset of $J_{i}$. Then the set $T={ }_{\operatorname{def}} U \backslash \bigcup_{i=1}^{t} S_{i}$ is polygonally connected. In fact, $T$ is an $L_{2}$-set (every two points of $T$ can be joined by a polygonal path in $T$ with at most two edges).

Proof Assume $a, b \in T$. If $[a, b] \subset T$, we are done. Otherwise define, for $i=$ $1, \ldots, t, A_{i}={ }_{\text {def }} \operatorname{aff}\left(J_{i} \cup\{a\}\right), B_{i}={ }_{\text {def }} \operatorname{aff}\left(J_{i} \cup\{b\}\right) . A_{i}$ and $B_{i}$ are flats of dimension $d-1$ or $d-2$.

Since $\operatorname{dim} U=d$, we can find a point $c \in U \backslash \bigcup_{i=1}^{t}\left(A_{i} \cup B_{i}\right)$. In fact, we can find such a point $c$ arbitrarily close to $\frac{1}{2}(a+b)$. If the line $\operatorname{aff}(a, c)$ meets $J_{i}$ at some point $x$ other than $a$, then $c \in \operatorname{aff}(a, x) \subset \operatorname{aff}\left(\{a\} \cup J_{i}\right)=A_{i}$, contrary to our choice of $c$. Therefore, $] a, c] \cap J_{i}=\emptyset$ for $i=1, \ldots, t$, hence $\left.] a, c\right] \subset U \backslash \bigcup_{i=1}^{t} J_{i} \subset$ $U \backslash \bigcup_{i=1}^{t} S_{i}=T$ (so $[a, c] \subset T$ ). The same argument, with $a$ and $A_{i}$ replaced by $b$ and $B_{i}$, shows that $[c, b] \subset T$.

Remark 2.1 Lemma 2.2 can be extended beyond the realm of convex sets, as follows:

If $U$ is an open connected set in $\mathbb{R}^{d}$, and $S_{1}, \ldots, S_{t}$ are subsets of $U$ with $\operatorname{dim}$ aff $S_{i} \leq d-2$ for $i=1, \ldots, t$, then the set $T=U \backslash \bigcup_{i=1}^{t} S_{i}$ is polygonally connected. If two points $a, b \in T$ can be joined by a polygonal path with $n$ edges within $S$, then they can be joined by a polygonal path with at most $\max (2, n)$ edges within $T$. The proof is similar.

\section{Polygonal Connectedness of int $\mathcal{C}$ and of $\operatorname{ext} \mathcal{C}$}

In this section we show that the open sets int $\mathcal{C}$ and $\operatorname{ext} \mathcal{C}$ are (polygonally) connected. For any two points $a, b \in \operatorname{int} \mathcal{C}$, we construct a polygonal path from $a$ to $b$ that lies entirely in int $\mathcal{C}$, stays mostly close to $|\mathcal{C}|$ and far from $\left|\operatorname{skel}_{d-3} \mathcal{C}\right|$. We do similarly for ext $\mathcal{C}$. We shall also bound from above the number of edges of the constructed paths. The bound will be approximately $\frac{n}{d}$, where $n={ }_{\text {def }} f_{d-1}(\mathcal{C})$ is the number of facets of $\mathcal{C}$.

\subsection{Some Preliminary Lemmata}

Let the facets of $\mathcal{C}$ be $F_{1}, \ldots, F_{n}\left(n=f_{d-1}(\mathcal{C})\right)$. For $i=1, \ldots, n$, let $u_{i}$ be a unit vector perpendicular to aff $F_{i}$. Choose the direction of $u_{i}$ in such a way that for each point $b \in \operatorname{relint} F_{i}$ and for all sufficiently small positive values of $\varepsilon, b+\varepsilon u_{i} \in \operatorname{ext} \mathcal{C}$ and $b-\varepsilon u_{i} \in \operatorname{int} \mathcal{C}$. If a subfacet $G$ of $\mathcal{C}$ is the intersection of $F_{i}$ and $F_{j}(1 \leq i<$ $j \leq n)$, define $u_{i j}=_{\text {def }} u_{i}+u_{j}$.

Lemma 3.1 If $g \in \operatorname{relint} G$ and $\varepsilon$ is a sufficiently small positive number, then $g+$ $\varepsilon u_{i j} \in \operatorname{ext} \mathcal{C}, g-\varepsilon u_{i j} \in \operatorname{int} \mathcal{C}$. 


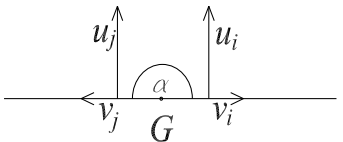

a

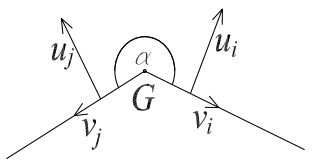

$\mathrm{b}$

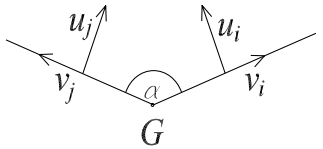

C

Fig. 3 Proof of Lemma 3.1

Proof The set aff $G$ is a $(d-2)$-flat. Denote by $A$ the $(d-2)$-dimensional linear subspace of $\mathbb{R}^{d}$ parallel to aff $G$. ( $A=\operatorname{aff} G-\operatorname{aff} G$ and aff $G=g+A$.) The facets $F_{i}$ and $F_{j}$ lie in two half-hyperplanes $H_{i}$ and $H_{j}$ bounded by aff $G$, say, $H_{i}=$ cone $_{g}\left(F_{i}\right)=\operatorname{aff} G+\mathbb{R}^{+} v_{i}, H_{j}=$ cone $_{g} F_{j}=\operatorname{aff} G+\mathbb{R}^{+} v_{j}$, where $v_{i}$ and $v_{j}$ are suitable unit vectors in the orthogonal complement $A^{+}$of $A$. If $\varepsilon$ is a sufficiently small positive number $\left(0<\varepsilon<\operatorname{dist}\left(g,\left|\mathcal{C} \backslash\left\{F_{i}, F_{j}, G\right\}\right|\right)\right)$, then $B^{d}(g, \varepsilon) \backslash|\mathcal{C}|=$ $B^{d}(g, \varepsilon) \backslash\left(H_{i} \cup H_{j}\right)$. The union $H_{i} \cup H_{j}$ divides $B^{d}(g, \varepsilon)$ into two open wedges, $B^{d}(g, \varepsilon) \cap \operatorname{int} \mathcal{C}$ and $B^{d}(g, \varepsilon) \cap \operatorname{ext} \mathcal{C}$. If $H_{i}$ and $H_{j}$ lie in the same hyperplane $\left(v_{j}=-v_{i}\right)$, then each of these two wedges is an open half-ball. In this case, $u_{i}=u_{j}$ (Fig. 3(a)), $u_{i j}=2 u_{i}=2 u_{j}$, and the lemma holds trivially. If $u_{i}$ and $u_{j}$ lie in different hyperplanes, then one of the wedges is larger than a half-ball, and the other is smaller.

In all cases we have

$$
\left\langle u_{i}, v_{j}\right\rangle=\left\langle u_{j}, v_{i}\right\rangle=\sin \alpha,
$$

where $\alpha$ is the dihedral angle of the wedge $B^{d}(g, \varepsilon) \cap \operatorname{ext} \mathcal{C}$ at $g$. If $\left\langle u_{i}, v_{j}\right\rangle<0$, then $B(g, \varepsilon) \cap \operatorname{ext} \mathcal{C}$ is the larger wedge (Fig. 3(b)), and if $\left\langle u_{i}, v_{j}\right\rangle>0$, then $B(g, \varepsilon) \cap \operatorname{int} \mathcal{C}$ is the larger wedge (Fig. 3(c)). Adding the equations $u_{i}=\left\langle u_{i}, u_{j}\right\rangle u_{j}+\left\langle u_{i}, v_{j}\right\rangle v_{j}$ and $u_{j}=\left\langle u_{j}, u_{i}\right\rangle u_{i}+\left\langle u_{j}, v_{i}\right\rangle v_{i}$ and using (2), we find that $\left(1-\left\langle u_{i}, u_{j}\right\rangle\right)\left(u_{i}+u_{j}\right)=$ $\sin \alpha\left(v_{i}+v_{j}\right)$.

If $u_{i} \neq u_{j}$, then $1-\left\langle u_{i}, u_{j}\right\rangle>0$, and therefore $u_{i j}=u_{i}+u_{j}=\frac{\sin \alpha}{1-\left\langle u_{i}, u_{j}\right\rangle} \times$ $\left(v_{i}+v_{j}\right)$. Thus $u_{i j}$ is a positive (resp., negative) multiple of $v_{i}+v_{j}$ when $\sin \alpha>0$ (resp., $\sin \alpha<0$ ). In both cases, $u_{i j}$ points towards $\operatorname{ext} \mathcal{C}$, and $-u_{i j}$ towards $\operatorname{int} \mathcal{C}$.

Lemma 3.2 ("Push away from $|\mathcal{C}|$ ")

(a) Suppose $F_{i}$ is a facet of $\mathcal{C}, G=F_{i} \cap F_{j}$ is a subfacet, $b \in \operatorname{relint} F_{i}, g \in \operatorname{relint} G$, and $u$ is a vector that satisfies $\left\langle u, u_{i}\right\rangle>0$. Define $I_{0}={ }_{\operatorname{def}}[b, g], I_{\varepsilon}={ }_{\operatorname{def}}[b+$ $\left.\varepsilon u, g+\varepsilon u_{i j}\right]\left(u_{i}, u_{j}\right.$ and $u_{i j}=u_{i}+u_{j}$ denote the same vectors as in the preceding lemma). If $\varepsilon$ is a sufficiently small positive number, then $I_{\varepsilon} \subset \operatorname{ext} \mathcal{C}, I_{-\varepsilon} \subset$ int $\mathcal{C}$. (The required smallness of $\varepsilon$ may depend on the choice of the points $b, g$ and of the vector $u$.)

(b) Suppose $F_{i}, F_{j}, F_{k}$ are distinct facets of $\mathcal{C}, G=F_{i} \cap F_{j}, H=F_{j} \cap F_{k}$ are subfacets $(G \neq H), g \in \operatorname{relint} G, h \in \operatorname{relint} H$. Define $J_{0}==_{\operatorname{def}}[g, h], J_{\varepsilon}={ }_{\operatorname{def}}$ $\left[g+\varepsilon u_{i j}, h+\varepsilon u_{j k}\right]$. If $\varepsilon>0$ is sufficiently small, then $J_{\varepsilon} \subset \operatorname{ext} \mathcal{C}$ and $J_{-\varepsilon} \operatorname{int} \mathcal{C}$.

Proof (a) First note that $I_{0}$ does not meet any facet of $\mathcal{C}$ except $F_{i}$ and $F_{j}$. The same holds for $I_{\varepsilon}$, provided $|\varepsilon|<\min \left(\frac{1}{2}, \frac{1}{\|u\|}\right) \cdot \operatorname{dist}\left(I_{0},\left|\mathcal{C} \backslash\left\{F_{i}, F_{j}, G\right\}\right|\right)$. By Lemma 3.1 
above, $g+\varepsilon u_{i j} \in \operatorname{ext} \mathcal{C}$ and $g-\varepsilon u_{i j} \in \operatorname{int} \mathcal{C}$, provided $\varepsilon$ is positive and sufficiently small. To complete the proof, it suffices to show that $I_{\varepsilon} \cap F_{i}=\emptyset$ and $I_{\varepsilon} \cap F_{j}=\emptyset$ (for sufficiently small $|\varepsilon|, \varepsilon \neq 0$ ).

As for $F_{i}:\left\langle u_{i}, u\right\rangle>0$ (given) and $\left\langle u_{i}, u_{i j}\right\rangle=1+\left\langle u_{i}, u_{j}\right\rangle>0$. Therefore, for any $\varepsilon \neq 0$ both endpoints of $I_{\varepsilon}$ lie (strictly) on the same side of the hyperplane aff $F_{i}$, hence $F_{i} \cap I_{\varepsilon}=\emptyset$.

As for $F_{j}$ : If $F_{j}$ and $F_{i}$ lie in the same hyperplane $\left(u_{i}=u_{j}\right)$, then the previous argument shows that $F_{j} \cap I_{\varepsilon}=\emptyset$ for all $\varepsilon \neq 0$ as well. If $u_{i} \neq u_{j}$, consider first the case where $\left\langle u_{i}, v_{j}\right\rangle<0$ (Fig. 3(b)). For $\varepsilon>0, I_{\varepsilon}$ lies in the open half-space $\left\{x \in \mathbb{R}^{d}:\left\langle u_{i}, x\right\rangle>\left\langle u_{i}, g\right\rangle\right\}$, whereas $F_{j}$ lies in the closed half-space $\left\{x \in \mathbb{R}^{d}:\left\langle u_{i}, x\right\rangle \leq\left\langle u_{i}, g\right\rangle\right\}$. Therefore, $I_{\varepsilon} \cap F_{j}=\emptyset$. For $\varepsilon<0,\left\langle u_{j}, g+\varepsilon u_{i j}\right\rangle=$ $\left\langle u_{j}, g\right\rangle+\varepsilon\left(1+\left\langle u_{i}, u_{j}\right\rangle\right)<\left\langle u_{j}, g\right\rangle$. On the other hand, $\left\langle u_{j}, b\right\rangle<\left\langle u_{j}, g\right\rangle$ (for any point $b \in \operatorname{relint} F_{i}$, since $\left.\left\langle u_{j}, v_{i}\right\rangle<0\right)$, and therefore $\left\langle u_{j}, b+\varepsilon u\right\rangle<\left\langle u_{j}, g\right\rangle$ for sufficiently small $|\varepsilon|, \varepsilon \neq 0$. Thus both endpoints of $I_{\varepsilon}$ lie on the same open side of the hyperplane aff $F_{j}$, hence $I_{\varepsilon} \cap F_{j}=\emptyset$.

In the case where $\left\langle u_{i}, v_{j}\right\rangle>0$ (Fig. 3(c) above), just repeat the previous argument with the roles of $\varepsilon>0$ and $\varepsilon<0$ interchanged.

(b) The proof is similar to that of (a). First, note that $J_{0}$ does not meet any facet of $\mathcal{C}$ except $F_{i}, F_{j}$, and $F_{k}$. The same holds for $J_{\varepsilon} \operatorname{provided}|\varepsilon|<\frac{1}{2} \cdot \operatorname{dist}\left(J_{0}, \mid \mathcal{C} \backslash\right.$ $\left.\left\{F_{i}, F_{j}, F_{k}, G, H\right\} \mid\right)$. By Lemma 3.1 above, $g+\varepsilon u_{i j}, h+\varepsilon u_{j k} \in \operatorname{ext} \mathcal{C}$ and $g-$ $\varepsilon u_{i j}, h-\varepsilon u_{j k} \in \operatorname{int} \mathcal{C}$, provided $\varepsilon$ is positive and sufficiently small. To complete the proof, it suffices to show that $J_{\varepsilon} \cap F_{i}=\emptyset, J_{\varepsilon} \cap F_{j}=\emptyset$, and $J_{\varepsilon} \cap F_{k}=\emptyset$ (for sufficiently small $|\varepsilon|, \varepsilon \neq 0$.

As for $F_{j}:\left\langle u_{j}, u_{i j}\right\rangle=1+\left\langle u_{j}, u_{i}\right\rangle>0$ and $\left\langle u_{j}, u_{j k}\right\rangle=1+\left\langle u_{j}, u_{k}\right\rangle>0$. Therefore, for any $\varepsilon \neq 0$, both endpoints of $J_{\varepsilon}$ lie on the same open side of the hyperplane aff $F_{j}$, hence $F_{j} \cap J_{\varepsilon}=\emptyset$.

As for $F_{i}$ : If $F_{j}$ and $F_{i}$ lie in the same hyperplane $\left(u_{i}=u_{j}\right)$, then the previous argument shows that $F_{i} \cap J_{\varepsilon}=\emptyset$ for all $\varepsilon \neq 0$ as well. If $u_{i} \neq u_{j}$, consider first the case $\left\langle u_{i}, v_{j}\right\rangle<0$ (Fig. 3(b) above). For $\varepsilon>0, J_{\varepsilon}$ lies in the open half-space $\{x \in$ $\left.\mathbb{R}^{d}:\left\langle u_{j}, x\right\rangle>\left\langle u_{j}, g\right\rangle\right\}$, whereas $F_{i}$ lies in the closed half-space $\left\{x \in \mathbb{R}^{d}:\left\langle u_{j}, x\right\rangle \leq\right.$ $\left.\left\langle u_{j}, g\right\rangle\right\}$. Therefore, $J_{\varepsilon} \cap F_{i}=\emptyset$. For $\varepsilon<0$, we have $\left\langle u_{i}, g+\varepsilon u_{i j}\right\rangle=\left\langle u_{i}, g\right\rangle+\varepsilon(1+$ $\left.\left\langle u_{i}, u_{j}\right\rangle\right)<\left\langle u_{i}, g\right\rangle$. On the other hand, $\left\langle u_{i}, h\right\rangle<\left\langle u_{i}, g\right\rangle$ (for any point $h \in F_{j} \backslash G$, since $\left\langle u_{i}, v_{j}\right\rangle<0$ ), and therefore $\left\langle u_{i}, h+\varepsilon u_{j k}\right\rangle<\left\langle u_{i}, g\right\rangle$ for sufficiently small $|\varepsilon|$. Thus both endpoints of $J_{\varepsilon}$ lie on the same open side of the hyperplane aff $F_{i}$, hence $J_{\varepsilon} \cap F_{i}=\emptyset$.

In the case where $\left\langle u_{i}, v_{j}\right\rangle>0$ (Fig. 3(c) above), just repeat the previous argument with the roles of $\varepsilon>0$ and $\varepsilon<0$ interchanged.

As for $F_{k}$ : Since the roles of $F_{i}$ and $F_{k}$ are interchangeable, the statement proved above for $F_{i}$ applies to $F_{k}$ as well.

Definition 3.1 Let $p$ be a point in $\mathbb{R}^{d} \backslash|\mathcal{C}|(=\operatorname{ext} \mathcal{C} \cup \operatorname{int} \mathcal{C})$, and $F$ be a facet of $\mathcal{C}$. We say that $p$ sees $F$ if, for some point $a \in \operatorname{relint} F,[p, a] \cap|\mathcal{C}|=\{a\}$.

Lemma 3.3 Assume $p \in \mathbb{R}^{d} \backslash|\mathcal{C}|$. Then $p$ sees at least one facet of $\mathcal{C}$.

Proof Assume, w.l.o.g., that $p \in \operatorname{ext} \mathcal{C}$. Let $q$ be a point in int $\mathcal{C}$. Let $U$ be a neighborhood of $q$ that lies entirely in int $\mathcal{C}$. Choose a point $q^{\prime} \in U$ such that the line aff $\left(p, q^{\prime}\right)$ 
does not meet any subfacet of $\mathcal{C}$. (This condition can be met by avoiding a finite number of hyperplanes through $p$.) Then the line segment $\left[p, q^{\prime}\right]$ must meet $|\mathcal{C}|$. Let $a$ be the first point of $|\mathcal{C}|$ on $\left[p, q^{\prime}\right]$ (starting from $p$ ). Then $a$ is a relative interior point of some facet $F$ of $\mathcal{C}$; and $[p, a] \cap|\mathcal{C}|=\{a\}$.

Definition 3.2 Let $p$ be a point in $\mathbb{R}^{d} \backslash|\mathcal{C}|$. We say that $p$ has a bounded horizon ("does not see the sky") with respect to $\mathcal{C}$ if every ray in $\mathbb{R}^{d}$ that emanates from $p$ meets $|\mathcal{C}|$. (This is always the case when $p \in \operatorname{int} \mathcal{C}$.) Otherwise we say that $p$ has an unbounded horizon. Of course, $p$ can be of unbounded horizon only if $p \in \operatorname{ext} \mathcal{C}$.

Proposition 3.1 If $p$ has a bounded horizon, then $p$ sees at least $d+1$ different facets of $\mathcal{C}$.

Proof By Lemma 3.3, $p$ sees at least one facet of $\mathcal{C}$. Assume we know that $p$ sees $k$ facets $F_{1}, \ldots, F_{k}$ of $\mathcal{C}, 1 \leq k \leq d$. Choose points $a_{i} \in$ relint $F_{i}, 1 \leq i \leq k$, such that $\left[p, a_{i}\right] \cap|\mathcal{C}|=\left\{a_{i}\right\}$, and unit vectors $u_{i}$ such that $\left\langle u_{i}, x\right\rangle=\left\langle u_{i}, a_{i}\right\rangle$ for all $x \in F_{i}$, and $\left\langle u_{i}, p\right\rangle<\left\langle u_{i}, a_{i}\right\rangle$. (If $p \in \operatorname{int} \mathcal{C}$, then our vectors $u_{i}$ are the same as the vectors $u_{i}$ defined before Lemma 3.1 above. If $p \in \operatorname{ext} \mathcal{C}$, then our vectors $u_{i}$ are the negatives of the vectors $u_{i}$ defined before Lemma 3.1.)

If $k<d$, or if $k=d$ and the vectors $u_{1}, \ldots, u_{d}$ are linearly dependent, let $w$ be a unit vector that is orthogonal to $u_{1}, \ldots, u_{k}$. If $k=d$ and the vectors $u_{1}, \ldots, u_{d}$ are linearly independent, let $w$ be a unit vector that satisfies $\left\langle u_{i}, w\right\rangle<0$ for $i=$ $1, \ldots, d$. By our choice of $w$, the ray $R(p, w)=\{p+\lambda w: \lambda \geq 0\}$ does not meet any of the facets $F_{1}, \ldots, F_{k}$. Since these facets are bounded, the same holds for the ray $R\left(p, w^{\prime}\right)$, provided $w^{\prime}$ is sufficiently close to $w$. Choose $w^{\prime}$ close to $w$ and such that the ray $R\left(p, w^{\prime}\right)$ does not meet any subfacet of $\mathcal{C}$. Let $a_{k+1}$ be the first point of $|\mathcal{C}|$ on $R\left(p, w^{\prime}\right)$. The point $a_{k+1}$ belongs to the relative interior of a facet $F_{k+1}$ of $\mathcal{C}$. Clearly, $p$ sees $F_{k+1}$, and $F_{k+1}$ is not one of the previous facets $F_{1}, \ldots, F_{k}$.

\section{$3.2 d$-Connectedness of the Dual Graph of $\mathcal{C}$}

Theorem 3.1 (Generalized Balinski-Klee Theorem) If $\mathcal{C}$ is $a(d-1)-P P M(d \geq 2)$, then the dual graph $D(\mathcal{C})$ of $\mathcal{C}$ is d-connected.

Remarks (1) For the definition of a $(d-1)-P P M$, see Definitions 1.2 and 1.3 above. Theorem 3.1 does not make use of the assumption that the complex $\mathcal{C}$ lies in $\mathbb{R}^{d}$; $\mathcal{C}$ may lie in $\mathbb{R}^{d^{\prime}}$ for some $d^{\prime}>d$. In fact, Theorem 3.1 can be stated for abstract polyhedral pseudomanifolds (that might, or might not be realizable).

(2) The theorem generalizes Balinski's Theorem on the $d$-connectedness of the graph (and of the dual graph) of a $d$-polytope (see [5] or [13, p. 213]). In fact, the proof given below uses Balinski's Theorem. For simplicial $(d-1)$-pseudomanifolds Theorem 3.1 was formulated and proved by V. Klee; see [16, Theorem 5].

Proof Note that if $d=2$, then the 1-PPM $\mathcal{C}$ is just a simple closed polygon, and the dual graph of $\mathcal{C}$ is a simple circuit, which is 2 -connected. Therefore, we assume in the sequel that $d \geq 3$. In the sequel we denote the dual graph of $\mathcal{C}$ by $D(\mathcal{C})$. 
Given two facets $A, B$ of $\mathcal{C}$, a path (of length $l$ ) that connects $A$ to $B$ in $D(\mathcal{C})$ is just a sequence $\mathcal{P}=\left(F_{0}, F_{1}, \ldots, F_{l}\right)$ of facets of $\mathcal{C}$ that satisfies: $F_{0}=A, F_{l}=B$, and $\operatorname{dim}\left(F_{i-1} \cap F_{i}\right)=d-2$ for $i=1, \ldots, l$. The facets $F_{1}, \ldots, F_{l-1}$ are the interior nodes of $\mathcal{P}$. The path $\mathcal{P}$ is simple if the facets $F_{0}, F_{1}, \ldots, F_{l}$ are all different. Since $D(\mathcal{C})$ is a graph of valence $\geq d$ at each node, it has at least $d+1$ nodes $\left(f_{d-1}(\mathcal{C}) \geq d+1\right)$, and by the Menger-Whitney Theorem the $d$-connectedness of $D(\mathcal{C})$ is equivalent to the following claim. (The Menger-Whitney Theorem can be formulated as follows (see [30, p. 171]): A graph is $k$-connected iff every pair of vertices can be joined by at least $k$ paths with pairwise disjoint interiors. The theorem goes back to K. Menger (see [21] and [22]) and, independently, H. Whitney [34]; see [33]. The theorem is used in the proof of Balinski's theorem; see [13, pp. 212-213], where it is attributed solely to Whitney, without mentioning his forerunner Menger.)

Claim 3.1 Given a list $A, B, C_{1}, \ldots, C_{d-1}$ of $d+1$ distinct facets of $\mathcal{C}$, there is a simple path $\mathcal{P}$ in $D(\mathcal{C})$ that connects $A$ to $B$ and avoids $C_{1}, \ldots, C_{d-1}$.

Proof of Claim The existence of a simple path $\mathcal{P}$ in $D(\mathcal{C})$ that connects $A$ to $B$ follows by the assumption that $D(\mathcal{C})$ is a connected graph (Condition (D) in Definition 1.3). To prove the claim above we proceed by induction. Assuming we have a simple path $\mathcal{P}$ in $D(\mathcal{C})$ that connects $A$ to $B$ and avoids $C_{1}, C_{2}, \ldots, C_{k-1}$ (for some $k, 1 \leq k \leq d-1$ ), we modify $\mathcal{P}$ to produce a simple path $\mathcal{P}^{\prime}$ that avoids $C_{k}$ as well. We shall need the following

Lemma 3.4 Let $J$ be a $(d-3)$-face of $\mathcal{C}$. The subgraph of $D(\mathcal{C})$ spanned by the facets of $\mathcal{C}$ that include $J$ is a disjoint union of simple circuits.

Proof of Lemma Every facet $(=(d-1)$-face $)$ of $\mathcal{C}$ that includes $J$ has exactly two subfacets $(=(d-2)$-faces) that include $J$. Conversely, every subfacet that includes $J$ is included in exactly two facets that include $J$ (consider the no branching Condition (C) in Definition 1.3). In other words, the subgraph under consideration is 2-regular. Since it is finite, it must be a disjoint union of simple circuits.

Back to the proof of Claim 3.1.

Induction step $k-1 \rightarrow k$. Let $\mathcal{P}=\left(F_{0}, F_{1}, \ldots, F_{l}\right)$ be a simple path in $D(\mathcal{C})$ that connects $A\left(=F_{0}\right)$ to $B\left(=F_{l}\right)$ and avoids $C_{1}, \ldots, C_{k-1}$ (for some $\left.1 \leq k \leq d-1\right)$. If $\mathcal{P}$ avoids $C_{k}$ as well, put $\mathcal{P}^{\prime}=\mathcal{P}$. Otherwise assume $C_{k}=F_{j}$ for some (unique) $j$ with $1 \leq j \leq l-1$.

Define $C={ }_{\text {def }} C_{k}=F_{j}, G={ }_{\text {def }} F_{j-1} \cap C, H={ }_{\text {def }} C \cap F_{j+1}$. In the dual graph of the $(d-1)$-polytope $C$ there are $d-1$ simple paths $\pi_{1}, \ldots, \pi_{d-1}$ with pairwise disjoint interiors that connect the $(d-2)$-faces $G$ and $H$. This follows from the $(d-1)$-connectedness of the dual graph of $C$ (Balinski's Theorem). Let $\pi=\left(\Phi_{0}, \Phi_{1}, \ldots, \Phi_{\lambda}\right)$ with $\Phi_{0}=G$ and $\Phi_{\lambda}=H$ be one of those $d-1$ paths $\left(\operatorname{dim}\left(\Phi_{i} \cap \Phi_{i+1}=d-3\right.\right.$ for $\left.0 \leq i \leq \lambda-1\right)$. $\pi$ induces a path $\Pi$ from $F_{j-1}$ to $F_{j+1}$ in $D(\mathcal{C})$ as follows. Each of the $(d-2)$-faces $\Phi_{i}(0 \leq i \leq \lambda)$ is included in exactly two $(d-1)$-faces of $\mathcal{C}$. One of them is $C$. Call the other one $Q_{i}$. In particular, $Q_{0}=F_{j-1}$ and $Q_{\lambda}=F_{j+1}$. For each $i, 0 \leq i<\lambda$, the three $(d-1)$-cells $Q_{i}, C$ and 
$Q_{i+1}$ share a $(d-3)$-face $\Psi_{i}={ }_{\text {def }} \Phi_{i} \cap \Phi_{i+1}$. Thus $\left\langle Q_{i}, C, Q_{i+1}\right\rangle$ is an arc of one of the circuits around $J={ }_{\operatorname{def}} \Psi_{i}$, as described in Lemma 3.4 above. The arc complementary to $\left\langle Q_{i}, C, Q_{i+1}\right\rangle$ within that circuit is an arc in $D(\mathcal{C})$ that connects $Q_{i}$ to $Q_{i+1}$, avoids $C$, and uses only $(d-1)$-cells that include $\Psi_{i}$. Concatenating these circumventing arcs from $i=0$ to $i=\lambda-1$, we obtain a path in $D(\mathcal{C})$ that connects $F_{j-1}$ to $F_{j+1}$, and avoids $C$. We call this path $\Pi$. Applying this procedure to the paths $\pi_{1}, \ldots, \pi_{d-1}$, we obtain $d-1$ paths $\Pi_{1}, \ldots, \Pi_{d-1}$ in $D(\mathcal{C})$ that connect $F_{j-1}$ to $F_{j+1}$ and avoid $C$.

Next we will show (Lemma 3.5 below) that two of these paths never share an internal node $\left(=(d-1)\right.$-cell). Since the list $C_{1}, \ldots, C_{d-1}$ of forbidden $(d-1)$ cells contains, apart from $C$, only $d-2$ additional cells, it follows that one of the avoiding paths, say $\Pi_{\nu}$, does not use any of the forbidden $(d-1)$-cells. Replace the arc $\left\langle F_{j-1}, C, F_{j}\right\rangle$ in $\mathcal{P}$ by $\Pi_{v}$, pass, if necessary, to a simple subpath, and call it $\mathcal{P}^{\prime}$. $\mathcal{P}^{\prime}$ is a simple path in $D(\mathcal{C})$ from $A$ to $B$ that avoids $C_{1}, \ldots, C_{k-1}$, and $C_{k}$.

Lemma 3.5 Let $\pi, \pi^{\prime}$ be two of the paths $\pi_{1}, \ldots, \pi_{d-1}$ listed above, that connect $G\left(=F_{j-1} \cap C\right)$ with $H\left(=F_{j+1} \cap C\right)$ in the dual graph of $C$ (say, $\pi=\pi_{m}, \pi^{\prime}=$ $\left.\pi_{m^{\prime}}, 1 \leq m<m^{\prime} \leq d-1\right)$. Let $\Pi$ and $\Pi^{\prime}$ be the paths induced by $\pi$ and $\pi^{\prime}$, respectively, that connect $F_{j-1}$ to $F_{j+1}$ in $D(\mathcal{C})$. Then $\Pi$ and $\Pi^{\prime}$ have no internal node in common.

Proof Assume $\pi=\left(\Phi_{0}, \Phi_{1}, \ldots, \Phi_{\lambda}\right)$, where $\Phi_{0}=F_{j-1} \cap C, \Phi_{\lambda}=F_{j+1} \cap C$, and each $\Phi_{i}$ is a $(d-2)$-face of $C$. Put $\Psi_{i}={ }_{\text {def }} \Phi_{i} \cap \Phi_{i+1}(i=0,1, \ldots, \lambda-1)$. Each $\Psi_{i}$ is a $(d-3)$-face of $C$. If $Q$ is an internal node of the induced path $\Pi$, then $Q \cap C$ is one of the $(d-2)$-cells $\Phi_{1}, \ldots, \Phi_{\lambda-1}$ or one of the $(d-3)$-cells $\Psi_{0}, \ldots, \Psi_{\lambda-1}$. The corresponding entities for $\pi^{\prime}$ are $\pi^{\prime}=\left(\Phi_{0}^{\prime}, \ldots, \Phi_{\lambda^{\prime}}^{\prime}\right)\left(\Phi_{0}^{\prime}=\Phi_{0}, \Phi_{\lambda^{\prime}}^{\prime}=\Phi_{\lambda}\right)$, and $\Psi_{i}^{\prime}=\Phi_{i}^{\prime} \cap \Phi_{i+1}^{\prime}\left(i=0,1, \ldots, \lambda^{\prime}-1\right)$.

If $Q^{\prime}$ is an internal node of $\Pi^{\prime}$, then $Q^{\prime} \cap C$ is one of the cells $\Phi_{1}^{\prime}, \ldots, \Phi_{\lambda^{\prime}-1}^{\prime}$ or $\Psi_{0}^{\prime}, \ldots, \Psi_{\lambda^{\prime}-1}^{\prime}$. To show that $Q \neq Q^{\prime}$, it suffices to check that the two sets $\left\{\Phi_{i}: 1 \leq\right.$ $i \leq \lambda-1\} \cup\left\{\Psi_{i}: 0 \leq i \leq \lambda-1\right\}$ and $\left\{\Phi_{i}^{\prime}: 1 \leq i \leq \lambda^{\prime}-1\right\} \cup\left\{\Psi_{i}^{\prime}: 0 \leq i \leq \lambda^{\prime}-1\right\}$ are disjoint. In fact, the $\Phi^{\prime}$-s are different from the $\Phi$-s, since $\pi$ and $\pi^{\prime}$ have no internal node in common. Each $\Psi_{i}$ is included in exactly two $(d-2)$-faces of $C$, which are adjacent nodes of $\pi$, and each $\Psi_{i^{\prime}}^{\prime}$ is included in exactly two $(d-2)$-faces of $C$, which are adjacent nodes of $\pi^{\prime}$. Since $\pi$ and $\pi^{\prime}$ have no internal nodes in common, this implies that $\Psi_{i} \neq \Psi_{i^{\prime}}^{\prime}$. Please pay attention to the possibility that, say, $\lambda=1$, and $\Psi_{0}=F_{j-1} \cap C \cap F_{j+1}$. In this case, we necessarily have $\lambda^{\prime}>1$, since $\pi^{\prime} \neq \pi$.

This concludes the proof of Claim 3.1 and ends the proof of Theorem 3.1.

\subsection{Upper Bounds for the Polygonal Diameter of int $\mathcal{C}$ and of $\operatorname{ext} \mathcal{C}$}

Definition 3.3 For a set $S \subset \mathbb{R}^{d}$ and points $a, b \in S$, denote by $\pi_{S}(a, b)$ the smallest number of edges of a polygonal line that connects $a$ to $b$ within $S .\left(\pi_{S}(a, b)={ }_{\mathrm{def}} \infty\right.$ if no such polygonal line exists.) If $S$ is polygonally connected, then $\pi_{S}(\cdot, \cdot)$ is an integer valued metric on $S$. The polygonal diameter of $S$ is defined as $\operatorname{poldiam}(S)=_{\mathrm{def}}$ 
$\sup \left\{\pi_{S}(a, b): a, b \in S\right\}$. Now we shall establish (almost) tight upper bounds for the polygonal diameter of int $\mathcal{C}$ and of $\operatorname{ext} \mathcal{C}$, where $\mathcal{C}$ is a $(d-1)-P P M$ in $\mathbb{R}^{d}$ with $n$ facets. In Sect. 4 below, we shall present examples of polyhedral $(d-1)$-spheres $\mathcal{C}, \mathcal{C}^{\prime}$ in $\mathbb{R}^{d}$ with $n$ facets (for all $d \geq 2$ and $d<n<\infty$ ) such that poldiam(int $\mathcal{C}$ ) = $\left\lfloor\frac{n}{d}\right\rfloor$, and poldiam $\left(\operatorname{ext} \mathcal{C}^{\prime}\right)=\left\lceil\frac{n}{d}\right\rceil$. For $d=2$, we shall even produce, in a subsequent paper, such examples with $\mathcal{C}=\mathcal{C}^{\prime}$ (see [25]). The following theorem clearly implies (F) in Sect. 1 above.

Theorem 3.2 (Upper bounds for poldiam $(\cdot))$ If $\mathcal{C}$ is a polyhedral $(d-1)$-pseudomanifold (i.e., $a(d-1)-P P M)$ with $n$ facets in $\mathbb{R}^{d}$, then the relations poldiam(int $\left.\mathcal{C}\right) \leq$ $2+\left\lfloor\frac{n-2}{d}\right\rfloor$ and poldiam $(\operatorname{ext} \mathcal{C}) \leq 3+\left\lfloor\frac{n-2}{d}\right\rfloor$ hold.

Proof Assume $a, b$ are two points in the same component (int $\mathcal{C}$ or ext $\mathcal{C}$ ) of $\mathbb{R} \backslash|\mathcal{C}|$. We shall distinguish three possible cases.

Case I: Both $a$ and $b$ are of bounded horizon (with respect to $\mathcal{C}$ ) (cf. Definition 3.2 above).

Case II: $a$ is of bounded horizon, whereas $b$ is of unbounded horizon (or vice versa).

Case III: Both $a$ and $b$ are of unbounded horizon.

Denote by $A$ (resp., $B$ ) the set of those facets $F$ of $\mathcal{C}$ such that $a$ (resp., $b$ ) sees a point in relint $F$ via $\mathbb{R}^{d} \backslash|\mathcal{C}|$. By Proposition 3.1 above, we know that $\# A \geq d+1$ if $a$ is of bounded horizon, and $\# A \geq 1$ otherwise (Lemma 3.3 above).

If $A \cap B \neq \emptyset$, say $F \in A \cap B$, then $a$ sees a point $a^{\prime} \in \operatorname{relint} F$ and $b$ sees a point $b^{\prime} \in$ relint $F$ via $\mathbb{R}^{d} \backslash|\mathcal{C}|$. Choose points $\left.a^{\prime \prime} \in\right] a, a^{\prime}$ [ (close to $a^{\prime}$ ) and $b^{\prime \prime} \in$ ]$b, b^{\prime}$ [ (close to $\left.b^{\prime}\right)$, such that the segment $\left[a^{\prime \prime}, b^{\prime \prime}\right]$ runs parallel to $F$ and misses $\mathcal{C}$. Then $\left\langle a, a^{\prime \prime}, b^{\prime \prime}, b\right\rangle$ is a 3 -path that connects $a$ to $b$ via $\mathbb{R}^{d} \backslash|\mathcal{C}|$. The upper bounds in Theorem 3.2 are all $\geq 3$, except for $\operatorname{int} \mathcal{C}$, when $n=d+1$. But when $n=d+1$, int $\mathcal{C}$ is the interior of a $d$-simplex, which is convex. Assume, from now on, that $A \cap B=\emptyset$, and consider each case separately.

Case I: In this case, \#A $\geq d+1, \# B \geq d+1$. Let $D=D(\mathcal{C})$ be the dual graph of $\mathcal{C}$. By Theorem 3.1, $D$ is $d$-connected. Extend $D$ to a graph $D^{\prime \prime}$ by adding two (auxiliary) vertices, $a$ and $b$, connecting $a$ by edges to all members of $A$ and $b$ to all members of $B$. As one can easily check, the extended graph $D^{\prime \prime}$ is again $d$ connected. Therefore, in $D^{\prime \prime}$ we can find $d$ paths $\pi_{1}, \ldots, \pi_{d}$ that connect $a$ to $b$ and have pairwise disjoint interiors. If we take these paths as short as possible (in particular, diagonal free), then each $\pi_{i}$ will visit the set $A$ (= neighbors of $a$ ) exactly once. Since $\# A \geq d+1$ and $\# B \geq d+1$, it follows that all these $d$ paths together visit at most $n-2$ vertices of $D$. The shortest of these paths, say $\pi_{1}$, is of the form $\left\langle a, F_{1}, \ldots, F_{m}, b\right\rangle$, where $m \leq\left\lfloor\frac{n-2}{d}\right\rfloor, F_{1}, \ldots, F_{m}$ are facets of $\mathcal{C}, F_{i-1}$ and $F_{i}$ share a subfacet $G_{i}$ for $i=2, \ldots, m, a$ sees via $\mathbb{R}^{d} \backslash|\mathcal{C}|$ a point $a^{\prime} \in \operatorname{relint} F_{1}$, and $b$ sees via $\mathbb{R}^{d} \backslash|\mathcal{C}|$ a point $b \in \operatorname{relint} F_{m}$. If we choose points $g_{i} \in \operatorname{relint} G_{i}$ for $i=2, \ldots, m$, then $\left\langle a, a^{\prime}, g_{2}, g_{3}, \ldots, g_{m}, b^{\prime}, b\right\rangle$ is a polygonal path of $m+2$ edges that connects $a$ to $b$ and runs along $|\mathcal{C}|$. By Lemma 3.2, this path can be pushed away from $|\mathcal{C}|$, thus producing a polygonal path of $m+2$ edges that connects $a$ to $b$ via $\mathbb{R}^{d} \backslash|\mathcal{C}|$.

Case II: In this case, extend the graph $D=D(\mathcal{C})$ to a graph $D^{\prime}$ by adding the (auxiliary) vertex $a$ and connecting $a$ by edges to all vertices in $A$. Fix a vertex 
$F^{\prime} \in B . D^{\prime}$ is again $d$-connected and contains $d$ paths $\pi_{1}, \ldots, \pi_{d}$ from $a$ to $F^{\prime}$, with pairwise disjoint interiors. If we take these $d$ paths to be diagonal free, then their union misses at least one vertex of $A$ (recall $\# A \geq d+1$ ). The union of their interiors misses $F^{\prime}$ as well, and thus uses at most $n-2$ facets of $\mathcal{C}$. The shortest of these paths, say $\pi_{1}$, is of the form $\left\langle a, F_{1}, \ldots, F_{m}, F^{\prime}\right\rangle$, where $m \leq\left\lfloor\frac{n-2}{d}\right\rfloor$. As in Case I, this path gives rise to a polygonal path $\left\langle a, a^{\prime}, g_{2}, \ldots, g_{m}, g_{m+1}, b^{\prime}, b\right\rangle$, with $a^{\prime} \in \operatorname{relint} F_{1}$, $g_{i} \in \operatorname{relint}\left(F_{i-1} \cap F_{i}\right)(i=2, \ldots, m), g_{m+1} \in \operatorname{relint}\left(F_{m} \cap F^{\prime}\right)$, and $b^{\prime} \in \operatorname{relint} F^{\prime}$, and thus to a polygonal path of at most $3+\left\lfloor\frac{n-2}{d}\right\rfloor$ edges that connects $a$ to $b$ via $\mathbb{R}^{d} \backslash|\mathcal{C}|$.

Case III: Denote by $U_{a}$ (resp., $U_{b}$ ) the set of unit vectors $u$ such that the ray $a+\mathbb{R}^{+} u$ (resp., $b+\mathbb{R}^{+} u$ ) misses $|\mathcal{C}|$. Since $|\mathcal{C}|$ is compact and $a \notin|\mathcal{C}|, U_{a}$ is an open subset of the unit sphere $S^{d-1} \subset \mathbb{R}^{d}$. We have $U_{a} \neq \emptyset$, since $a$ is of unbounded horizon, and the same holds for $U_{b}$. Choose vectors $u_{a} \in U_{a}$ and $u_{b} \in U_{b}$ such that $u_{b} \neq-u_{a}$. There is a linear function $f$ that satisfies $f\left(u_{a}\right)=f\left(u_{b}\right)=1$. Put $M={ }_{\mathrm{def}}$ $\max \{f(x): x \in|\mathcal{C}|\}$. If $t>M-\min \{f(a), f(b)\}$, then the segment $\left[a+t u_{a}, b+t u_{b}\right]$ misses $|\mathcal{C}|$, and $\left\langle a, a+t u_{a}, b+t u_{b}, b\right\rangle$ is a polygonal path of three edges that connects $a$ to $b$ via $\mathbb{R}^{d} \backslash|\mathcal{C}|$.

\section{Lower Bounds for the Maximal Polygonal Diameter of the Interior and Exterior of a $(d-1)-P P M$ in $\mathbb{R}^{d}$}

For $n \geq d+1>0$, define (note that poldiam(.) is defined in Definition 3.3 above):

$\operatorname{pd}-\operatorname{int}(d, n)=_{\text {def }} \max \left\{\operatorname{poldiam}(\operatorname{int} \mathcal{P}): \mathcal{P}\right.$ is a $(d-1)-P P M$ in $\mathbb{R}^{d}$ and $\left.f_{d-1}(\mathcal{P})=n\right\}$

("pd" stands of "polygonal diameter") and

$$
\begin{gathered}
\operatorname{pd}-\operatorname{ext}(d, n)=\text { def } \max \left\{\operatorname{poldiam}(\operatorname{ext} \mathcal{P}): \mathcal{P} \text { is a }(d-1)-P P M \text { in } \mathbb{R}^{d}\right. \\
\text { and } \left.f_{d-1}(\mathcal{P})=n\right\} .
\end{gathered}
$$

The following upper bounds were established in Theorem 3.2 above:

$$
\begin{aligned}
& \operatorname{pd}-\operatorname{int}(d, n) \leq 2+\left\lfloor\frac{n-2}{d}\right\rfloor, \\
& \operatorname{pd-ext}(d, n) \leq 3+\left\lfloor\frac{n-2}{d}\right\rfloor .
\end{aligned}
$$

In this section we produce two $(d-1)-P P M s \mathcal{P}$ and $\mathcal{Q}$ in $\mathbb{R}^{d}$, with $f_{d-1}(\mathcal{P})=$ $f_{d-1}(\mathcal{Q})=n, n \geq d+1>0$, such that poldiam $(\operatorname{int} \mathcal{P})=\left\lfloor\frac{n}{d}\right\rfloor$ and poldiam $(\operatorname{ext} \mathcal{Q})=$ $\left\lceil\frac{n}{d}\right\rceil$. These examples establish lower bounds $\left\lfloor\frac{n}{d}\right\rfloor$ (resp., $\left\lceil\frac{n}{d}\right\rceil$ ) for $\operatorname{pd}-\operatorname{int}(d, n)$ (resp., $\operatorname{pd}$-ext $(d, n))$ which are quite close to the upper bounds given in (3) (resp., (4)) above.

Example 4.1 (Zigzag sleeve) This is an example of a polyhedral $(d-1)$-sphere $\mathcal{P}$ in $\mathbb{R}^{d}$ (i.e., combinatorially equivalent to the boundary complex of a convex polytope in $\left.\mathbb{R}^{d}\right)$ with $n(\geq d+1>0)$ facets such that poldiam(int $\left.\mathcal{P}\right)=m=\operatorname{def}\left\lfloor\frac{n}{d}\right\rfloor$. Assume first that $n \equiv 0(\bmod d)$, i.e., $n=m d, m \geq 2$. Let $\Delta^{d-1}$ be a $(d-1)$-simplex in $\mathbb{R}^{d}$ 
Fig. 4 Example 4.1 for $m=5$, $d=3$

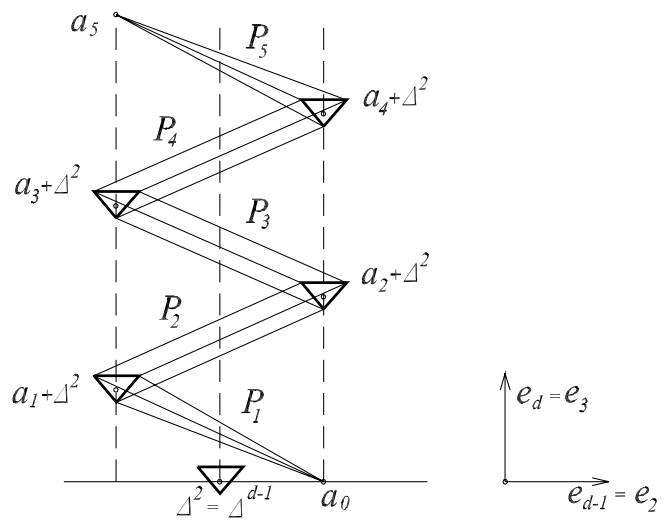

with barycenter at the origin $o$ contained in the relative interior of the unit $(d-1)$ ball $B^{d-1}(o, 1)$ of $\mathbb{R}^{d-1}$, where $\mathbb{R}^{d-1}={ }_{\text {def }}\left\{x=\left(\xi_{1}, \ldots, \xi_{d}\right) \in \mathbb{R}^{d}: \xi_{d}=0\right\}$, and let $F_{1}, \ldots, F_{d}$ be the facets of $\Delta^{d-1}$. Define $m+1$ points $a_{0}, a_{1}, \ldots, a_{m}$ by $a_{v}={ }_{\text {def }}$ $(-1)^{v} e_{d-1}+v e_{d}(v=0,1, \ldots, m)$. For $v=1, \ldots, m$, define a $d$-polytope $P_{v}$ as follows:

$$
\begin{aligned}
& P_{1}={ }_{\text {def }} \operatorname{conv}\left(\left\{a_{0}\right\} \cup\left(a_{1}+\Delta^{d-1}\right)\right), \\
& P_{v}={ }_{\text {def }} \operatorname{conv}\left(\left(a_{v-1}+\Delta^{d-1}\right) \cup\left(a_{v}+\Delta^{d-1}\right)\right)=\left[a_{v-1}, a_{v}\right]+\Delta^{d-1}
\end{aligned}
$$

for $2 \leq v<m$, and for $v=m$ we define $P_{m}=\operatorname{def}\left(\operatorname{conv}\left(a_{m-1}+\Delta^{d-1}\right) \cup\left\{a_{m}\right\}\right)$. Define also $P={ }_{\text {def }} \bigcup_{v=1}^{m} P_{v} . P_{1}$ is a $d$-simplex (a $d$-pyramid based on $a_{1}+\Delta^{d-1}$ apexed at $\left.a_{0}\right), P_{v}, 1<v<m$, is a $d$-prism on a $(d-1)$-simplex (the sum of the segment $\left[a_{v-1}, a_{v}\right]$ and the simplex $\Delta^{d-1}$ ), and $P_{m}$ is a $d$-simplex (a $d$-pyramid based on $a_{m-1}+\Delta^{d-1}$ and apexed at $a_{m}$ ). The union $P=P_{1} \cup P_{2} \cup \cdots \cup P_{m}$ is piecewise linearly homeomorphic (as a transformation that changes the $d$-coordinate only, i.e., a "piecewise shear" parallel to $\left.\mathbb{R}^{d-1}\right)$ to a convex polytope which is a $(d-1)$-prism on a $(d-1)$-simplex with two $d$-simplices attached to its two bases, like a pencil sharpened at both ends (see Fig. 4 for $m=5, d=3$ ). (Such a convex polytope may be called a bi-apexed prism.)

The boundary complex $\mathcal{P}$ of $P$ consists of $m d(=n)$ convex $(d-1)$-polytopes $(2 d(d-1)$-simplices and $(m-2) \cdot d(d-1)$-prisms on $(d-2)$-simplices $)$, listed as follows:

$$
\begin{aligned}
& \operatorname{conv}\left(\left\{a_{0}\right\} \cup\left(a_{1}+F_{i}\right)\right), \quad\left[a_{\nu-1}, a_{\nu}\right]+F_{i} \quad(2 \leq v \leq m-1), \\
& \operatorname{conv}\left(\left(a_{m-1}+F_{i}\right) \cup\left\{a_{m}\right\}\right),
\end{aligned}
$$

all this for $i=1,2, \ldots, d$. All these $(d-1)$-polytopes, together with all their faces, form a $(d-1)-P P M$, henceforth denoted by $\mathcal{P}$, satisfying int $\mathcal{P}=\operatorname{int} P$ (here int $\mathcal{P}$ is the interior of $\mathcal{P}$ in the sense of the Jordan-Brouwer separation theorem (Theorem 1.1 above) and int $P$ is the (topological) interior of $P$ as a (closed) set in $\mathbb{R}^{d}$ ). The following proposition implies that poldiam $($ int $\mathcal{P})=m$. 
Proposition 4.1 It is possible to proceed from any point $q_{0} \in \operatorname{int} B^{d}\left(a_{0}, 1\right) \cap$ int $P$ (a 1-vicinity of $a_{0}$ in int $P$ ) to any point $q_{m} \in \operatorname{int} B^{d}\left(a_{m}, 1\right) \cap$ int $P$ (a 1-vicinity of $a_{m}$ in int $P$ ) by a polygonal path of $m$ edges, but not by less than this.

Proof Define $\gamma=$ def $\left\langle q_{0}, a_{1}, a_{2}, \ldots, a_{m-1}, q_{m}\right\rangle \cdot \gamma$ is a polygonal path of $m$ edges in int $P$ from $q_{0}$ to $q_{m}$. This proves the first part of the proposition. To prove the second part, we give first an informal argument (see Fig. 4). In order to reach from $q_{0}$ to $P_{1} \cap P_{2}\left(=a_{1}+\Delta^{d-1}\right)$, there must be a decreasing segment $\left(\xi_{d-1}\right.$ decreases as a function of $\xi_{d}$ ); in order to reach from $P_{1} \cap P_{2}$ to $P_{2} \cap P_{3}\left(=a_{2}+\Delta^{d-1}\right)$, there must be an increasing segment ( $\xi_{d-1}$ increases as a function of $\xi_{d}$ ), etc.; finally, in order to reach from $P_{m-1} \cap P_{m}$ to $q_{m}$, there must be a decreasing [increasing] segment if $m$ is odd [if $m$ is even]. This makes a zigzag of $m$ edges.

Formal proof. Among the polygonal paths from $q_{0}$ to $q_{m}$ in int $P$, let $\gamma$ be a polygonal path of minimal number, say $n$, of edges, and let $p_{0}, p_{1}, \ldots, p_{n}$ be its vertices in this order, i.e., $\gamma=\left\langle p_{0}=q_{0}, p_{1}, p_{2}, \ldots, p_{n-1}, p_{n}=q_{m}\right\rangle$. (We have to show that $n \geq m$.) Note that the role played by $n$ here is different from that played by $n={ }_{\mathrm{def}}$ $f_{d-1}(\mathcal{C})$ defined supra.

We will think of $\gamma$ as a (piecewise linear and continuous) function from [0,1] to $\mathbb{R}^{d}$ with $\gamma(0)=q_{0}$ and $\gamma(1)=q_{m}$. Since $\gamma(0)=q_{0} \neq q_{m}=\gamma(1), \gamma$ is not a circuit.

Claim $4.1 \gamma$ is 1-1, i.e., $\gamma$ is a simple path.

Proof of Claim If $\gamma$ is not 1-1, then it contains a circuit, say $\tilde{\gamma}:\left[t_{1}, t_{2}\right] \rightarrow \mathbb{R}^{d}$ with $\left[t_{1}, t_{2}\right] \subset[0,1], \gamma(t)=\tilde{\gamma}(t)$ for $t \in\left[t_{1}, t_{2}\right]$ and $\tilde{\gamma}\left(t_{1}\right)=\tilde{\gamma}\left(t_{2}\right)$. Reduce $\gamma$ to $\left[0, t_{1}\right] \cup$ $\left[t_{2}, 1\right]$ (i.e., drop the circuit $\tilde{\gamma}$ from $\gamma$ ). The resulting polygonal path connects $q_{0}$ to $q_{m}$ via int $P$ and it clearly has fewer edges then $\gamma$, a contradiction. This proves Claim 4.1.

For $1 \leq i \leq m-1$, define a vertex $q_{i}$ of $\gamma$ as follows: Put $t_{i}=_{\operatorname{def}} \max \{t$ with $\left.0 \leq t \leq 1: \gamma_{d}(t)=i\right\}\left(t_{i}\right.$ is the last time that $\gamma$ visits the hyperplane $\left.\xi_{d}=i\right)$. Clearly, $\gamma\left(t_{i}\right) \in a_{i}+\Delta^{d-1}\left(=P_{i} \cap P_{i+1}\right)$, hence

$$
\operatorname{sg}\left(\gamma_{d-1}\left(t_{i}\right)\right)=(-1)^{i}, \quad 1 \leq i \leq m-1,
$$

and by Claim 4.1 above, $\gamma\left(t_{i}\right) \in\left[p_{\iota-1}, p_{\iota}[\right.$ for some unique $\iota=\iota(i), 1 \leq \iota \leq n$. Define

$$
q_{i}=\operatorname{def} \begin{cases}p_{\iota-1} & \text { if } \operatorname{sg}\left(\left(p_{\iota-1}\right)_{d-1}\right)=(-1)^{i}, \\ p_{\iota} & \text { otherwise. }\end{cases}
$$

This definition ensures that

$$
\operatorname{sg}\left(\left(q_{i}\right)_{d-1}\right)=(-1)^{i}\left(=\operatorname{sg}\left(a_{i}\right)\right)
$$

for $0 \leq i \leq m$. We will show that the vertices $q_{i}, 0 \leq i \leq m$, are all different.

Lemma 4.1 Let $[u, v]$ be a segment contained in int $P$ such that $(u)_{d}=i$ for some $i$ with $1 \leq i \leq m-1,(v)_{d} \neq i$ and $[u, v] \cap G=\emptyset$, where $G=_{\operatorname{def}}\left\{x \in \mathbb{R}^{d}:(x)_{d-1}=0\right\}$. Then either $i-1<(v)_{d}<i$ or $i<(v)_{d}<i+1$. 
Proof of Lemma Put $G_{i}={ }_{\operatorname{def}}\left\{x \in \mathbb{R}^{d}: \operatorname{sg}(x)_{d-1}=(-1)^{i}\right.$ or 0$\}$. Note that $u \in a_{i}+$ $\Delta^{d-1}\left(=P_{i} \cap P_{i+1}\right)$, hence $G_{i}$ is the closed half-space bounded by $G$ which contains $u$ (and $v$ ).

The $d$-polytope $P_{i}\left[P_{i+1}\right]$ is a $d$-prism if $1<i \leq m-1[1 \leq i<m-1]$, a $d$ simplex if $i=1[i=m-1]$; and it is contained in the layer $L_{i}^{-}={ }_{\operatorname{def}}\left\{x \in \mathbb{R}^{d}: i-\right.$ $\left.1 \leq(x)_{d} \leq i\right\}\left[L_{i}^{+}={ }_{\text {def }}\left\{x \in \mathbb{R}^{d}: i \leq(x)_{d} \leq i+1\right\}\right]$. The $d$-polytope $Q_{i}={ }_{\text {def }} P_{i} \cap$ $G_{i}\left[Q_{i+1}=_{\text {def }} P_{i+1} \cap G_{i}\right]$ satisfies

$$
\text { int } Q_{i} \subset \operatorname{int} L_{i}^{-} \quad\left[\operatorname{int} Q_{i+1} \subset \operatorname{int} L_{i}^{+}\right] \text {, }
$$

and it has $d+2$ facets: $d$ facets are subsets of the $d$ facets of $P_{i}\left[P_{i+1}\right]$ whose relative interiors are contained in int $L_{i}^{-}$[int $\left.L_{i}^{+}\right]$, henceforth called loxo facets, as well as the two facets $a_{i}+\Delta^{d-1}\left(=P_{i} \cap P_{i+1}\right)$ and $G \cap P_{i}\left[G \cap P_{i+1}\right]$. If $(v)_{d}<i\left[i<(v)_{d}\right]$, then $\left.\left.] u, v] \cap \operatorname{int} Q_{i} \neq \emptyset[] u, v\right] \cap \operatorname{int} Q_{i+1} \neq \emptyset\right]$, and since ]u,v] does not intersect any of the $d$ loxo facets of $P_{i}\left[P_{i+1}\right]$ (since $[u, v] \subset$ int $P$ ) and it does not intersect the facet $G \cap P_{i}\left[G \cap P_{i+1}\right]$ (since $[u, v] \cap G=\emptyset$ ), it follows that $[u, v] \cap$ bd $Q_{i}=$ $u\left[[u, v] \cap\right.$ bd $\left.Q_{i+1}=u\right]$, hence $v \in$ int $Q_{i}\left[v \in\right.$ int $\left.Q_{i+1}\right]$. It follows from (7) that $i-1<(v)_{d}<i$ if $(v)_{d}<i\left[i<(v)_{d}<i+1\right.$ if $\left.i<(v)_{d}\right]$. This proves Lemma 4.1.

Claim 4.2 $\left(q_{0}\right)_{d}<1, m-1<\left(q_{m}\right)_{d}$ and for $1 \leq i \leq m-1, i-1<\left(q_{i}\right)_{d}<i+1$.

Proof of Claim The first two inequalities follow from $\left\|q_{0}-a_{0}\right\|<1$ and $\left\|q_{m}-a_{m}\right\|<$ 1 , respectively. Let $1 \leq i \leq m-1$. If $\left(q_{i}\right)_{d}=i$, we are done. Assume $\left(q_{i}\right)_{d} \neq i$. Using the notation introduced above, $\left\{\gamma\left(t_{i}\right), q_{i}\right\} \subset$ int $G_{i}$ by (5) and (6), hence $\left[\gamma\left(t_{i}\right), q_{i}\right] \cap$ $G=\emptyset$. Since $\gamma\left(t_{i}\right) \in P_{i} \cap P_{i+1}$, the segment $\left[\gamma\left(t_{i}\right), q_{i}\right]$ satisfies the conditions of Lemma 4.1 above (with $u=\gamma\left(t_{i}\right)$ ) and $v=q_{i}$ ), hence either $i-1<\left(q_{i}\right)_{d}<i$ or $i<\left(q_{i}\right)_{d}<i+1$, hence $i-1<\left(q_{i}\right)_{d}<i+1$. This proves Claim 4.2.

We show now that $q_{i} \neq q_{j}$ for $0 \leq i<j \leq m$. If $j$ and $i$ are of different parities, this follows from (6). Let $j=i+2 \kappa$ for some $\kappa \geq 1$. In view of Claim 4.2,

$$
\left(q_{i}\right)_{d}<i+1<\left(q_{i+2}\right)_{d}<i+3<\left(q_{i+4}\right)_{d}<\cdots<i+(2 \kappa-1)<\left(q_{i+2 \kappa}\right)_{d},
$$

hence $q_{i} \neq q_{i+2 \kappa}\left(=q_{j}\right)$. This concludes the proof of Proposition 4.1.

It remains to modify Example 4.1 to the case where $n \not \equiv 0(\bmod d)$, i.e., $n=$ $m d+r, 1 \leq r \leq d-1$. If $r=1$, truncate the vertex $a_{m}$ of $P$ by a hyperplane passing in an $\varepsilon$-vicinity of $a_{m}$ for some sufficiently small $\varepsilon>0$. The resulting body $P^{\prime}$ has $m d+1(=n)$ facets, $d$ new vertices and, clearly, if $\varepsilon$ is small enough, then poldiam (int $P^{\prime}$ ) $=$ poldiam $($ int $P)=m=\left\lfloor\frac{n}{d}\right\rfloor$. If $r=2$, truncate $P$ near $a_{m}$ as above, and then truncate $P^{\prime}$ near enough to one of its new vertices; the resulting body $P^{\prime \prime}$ has $m d+2(=n)$ facets and again poldiam(int $\left.P^{\prime \prime}\right)=m=\left\lfloor\frac{n}{d}\right\rfloor$. For $3 \leq r \leq d-1$ make, similarly, $r$ truncations near $a_{m}$ to obtain a body $P^{(r)}$ with $m d+r(=n)$ facets and poldiam $\left(\right.$ int $\left.P^{(r)}\right)=m=\left\lfloor\frac{n}{d}\right\rfloor$.

Example 4.2 (Pyramid with a zigzag cave) Now we will modify the complex of Example 4.1 above to obtain a $(d-1)-P P M \mathcal{Q}$ in $\mathbb{R}^{d}$ with $n(\geq d+1>0)$ facets 
such that poldiam $(\operatorname{ext} \mathcal{Q})=\left\lceil\frac{n}{d}\right\rceil$. Again assume first that $n \equiv 0(\bmod d)$, i.e., $n=m d$ $(m \geq 2)$. In this case, $\left\lceil\frac{n}{d}\right\rceil=m$, so $\mathcal{Q}$ will have $m d$ facets, and $\operatorname{poldiam}(\mathcal{Q})=m$.

Define $m d$-polytopes $Q_{1}, \ldots, Q_{m}$ as follows:

(a) $Q_{v}={ }_{\mathrm{def}} P_{v}$ for $4 \leq v \leq m$.

(b) $Q_{2}={ }_{\mathrm{def}} \operatorname{conv}\left(\left(e_{d}+M \cdot \Delta^{d-1}\right) \cup\left(a_{2}(\varepsilon)+\Delta^{d-1}\right)\right)$ with large enough $M$ satisfying $\pm e_{d-1}+\left(e_{d}+\Delta^{d-1}\right) \subset \operatorname{relint}\left(e_{d}+M \cdot \Delta^{d-1}\right)\left(\subset H={ }_{\operatorname{def}}\left\{x \in \mathbb{R}^{d}:(x)_{d}=1\right\}\right)$,

and $a_{2}(\varepsilon)={ }_{\text {def }} e_{d-1}+(3-\varepsilon) e_{d}$, where $\varepsilon$ is a positive number whose smallness is determined below. Since $M \cdot \Delta^{d-1}$ is a homothetic image of $\Delta^{d-1}$ and $a_{2}(\varepsilon)+$ $\Delta^{d-1}$ is a translate of $\Delta^{d-1}$, an easy calculation shows that $e_{d}+M \cdot \Delta^{d-1}$ is a homothet of $a_{2}(\varepsilon)+\Delta^{d-1}$ with center of homothety $b=\operatorname{def} \frac{a_{2}(\varepsilon)-\frac{1}{M} e_{d}}{1-\frac{1}{M}}$ (and constant of homothety $M)$. Hence $Q_{2}$ is combinatorially equivalent to a $d$-prism on a $(d-1)$-simplex (same as $\left.\operatorname{conv}\left(\left(a_{1}+\Delta^{d-1}\right) \cup\left(a_{2}(\varepsilon)+\Delta^{d-1}\right)\right)\right)$. The facets $G_{i}={ }_{\text {def }} \operatorname{conv}\left(\left(e_{d}+M F_{i}\right) \cup\left(a_{2}(\varepsilon)+F_{i}\right)\right), 1 \leq i \leq d$, of $Q_{2}$ (recall that $F_{i}, 1 \leq$ $i \leq d$, are facets of $\Delta^{d-1}$ ) will be referred to below as the side facets of $Q_{2}$.

(c) $Q_{3}={ }_{\text {def }} \operatorname{conv}\left(\left(a_{2}(\varepsilon)+\Delta^{d-1}\right) \cup\left(a_{3}+\Delta^{d-1}\right)\right) . Q_{3}$ is a $d$-prism on a $(d-1)$ simplex analogous to $P_{3}$ above, where $a_{2}(\varepsilon)$ plays the role of $a_{2}$ in the definition of $P_{3}$. The replacement of $a_{2}$ by $a_{2}(\varepsilon)$ (where $\varepsilon>0$ is small) flattens $Q_{3}$ in the $d$-axis direction (the $d$-axis difference between the two bases of the prism is small).

(d) $Q_{1}={ }_{\text {def }} \operatorname{conv}\left(\left(e_{d}+M \cdot \Delta^{d-1}\right) \cup\left\{a_{0}(L)\right\}\right)$, where $a_{0}(L)={ }_{\text {def }}(L+1) e_{d}$ and $L$ is a positive number whose largeness is determined below.

First we determine $M$. Since $\Delta^{d-1} \subset$ relint $B^{d-1}(o, 1)$ (= the relative interior of the unit $(d-1)$-ball centered at the origin $o$ in the plane $\left.(x)_{d}=0\right)$ and since $o \in \operatorname{relint} \Delta^{d-1}$,

$$
\pm e_{d-1}+\Delta^{d-1} \subset \text { relint } B^{d-1}(o, 2)
$$

(= the relative interior of the $(d-1)$-ball of radius 2 centered in the origin in the hyperplane $\left.(x)_{d}=0\right)$. Clearly, $B^{d-1}(o, 2) \subset M \cdot \Delta^{d-1}$ for

$$
M>\frac{2}{\operatorname{dist}\left(o, \operatorname{relbd} \Delta^{d-1}\right)},
$$

hence (8) follows from (9) provided $M$ satisfies (10). So put

$$
M={ }_{\operatorname{def}} \frac{2}{\operatorname{dist}\left(o, \operatorname{relbd} \Delta^{d-1}\right)}+1 .
$$

Next we determine $\varepsilon$. (As mentioned above, the smallness of $\varepsilon$ determines the flatness of $Q_{3}$ in the $d$-axis direction.)

Put $H={ }_{\text {def }}\left\{x \in \mathbb{R}^{d}:(x)_{d}=1\right\}, H^{-}={ }_{\operatorname{def}}\left\{x \in \mathbb{R}^{d}:(x)_{d} \leq 1\right\}$ and $\hat{H}^{-}=$ (cone $\left._{b}\left(e_{d}+M \cdot \Delta^{d-1}\right)\right) \cap$ int $H^{-}$. Our main concern in determining $\varepsilon>0$ is

Requirement 4.1 (Smallness of $\varepsilon>0$ ) For $u \in \hat{H}^{-}$and $z^{+} \in a_{3}+\Delta^{d-1}$ the half open segment $\left[u, z^{+}\right.$[ avoids the basis $a_{2}(\varepsilon)+\Delta^{d-1}$ of $Q_{3}$. In other words, it is 
impossible to reach from a point in $\hat{H}^{-}$a point in the upper basis $a_{3}+\Delta^{d-1}$ of $Q_{3}$ directly by a segment via the lower basis $a_{2}(\varepsilon)+\Delta^{d-1}$ of $Q_{3}$.

We will show now the existence of such a (small) positive $\varepsilon$.

Put $G==_{\text {def }}\left\{x \in \mathbb{R}^{d}:(x)_{d-1}=0\right\}, G_{-}==_{\operatorname{def}}\left\{x \in \mathbb{R}^{d}:(x)_{d-1} \leq 0\right\}, G_{+}==_{\operatorname{def}}\{x \in$ $\left.\mathbb{R}^{d}:(x)_{d-1} \geq 0\right\}$, and $H_{3}={ }_{\text {def }}\left\{x \in \mathbb{R}^{d}:(x)_{d}=3\right\}$.

Since $a_{3}+\Delta^{d-1} \subset$ int $G_{-}$, Requirement 4.1 will be met provided

$$
\left(\operatorname{relint}\left(\operatorname{cone}_{u}\left(z^{-}\right)\right)\right) \cap H_{3} \subset G_{+}
$$

for $u \in \hat{H}^{-}$and $z^{-} \in a_{2}(\varepsilon)+\Delta^{d-1}$. Consider the (parallel) $(d-2)$-flats $F_{1}^{d-2}={ }_{\mathrm{def}}$ $3 e_{d}+\operatorname{span}\left(e_{1}, e_{2}, \ldots, e_{d-2}\right)$ and $F_{0}^{d-2}={ }_{\operatorname{def}} e_{d}+(M+1) e_{d-1}+\operatorname{span}\left(e_{1}, e_{2}\right.$, $\left.\ldots, e_{d-2}\right)$.

We have $F_{0}^{d-2} \| F_{1}^{d-2}, F_{1}^{d-2} \subset H_{3} \cap G$ and $F_{0}^{d-2} \subset H . F_{0}^{d-2}$ is a hyperplane of $H$ strictly supporting $e_{d}+M \cdot \Delta^{d-1}(\subset H)$. Put $I^{d-1}={ }_{\operatorname{def}} \operatorname{aff}\left(F_{0}^{d-2} \cup F_{1}^{d-2}\right)$. The hyperplane $I^{d-1}$ is the common boundary of two closed half-spaces $I_{-}^{d-1}, I_{+}^{d-1}$; choose the signs,+- so that $e_{d}+M \cdot \Delta^{d-1} \subset I_{-}^{d-1}$, so $H_{3} \cap G_{+} \subset I_{+}^{d-1}$. Define $\delta={ }_{\text {def }} \operatorname{dist}\left(e_{d-1}+\Delta^{d-1}, G\right)$; since the two sets involved in this definition are disjoint, $e_{d-1}+\Delta^{d-1}$ is compact and $G$ is closed, $\delta>0$. Let $\varepsilon>0$ satisfy

$$
\frac{\varepsilon}{\delta}<\frac{2}{M+1} .
$$

The difference between $F_{0}^{d-2}$ and $F_{1}^{d-2}$ in the $d$-axis direction is 2 , and their difference in the $(d-1)$-axis direction is $M+1$. It follows that if $\varepsilon$ satisfies (12), then $a_{2}(\varepsilon)+\Delta^{d-1} \subset I_{+}^{d-1}$, and Condition (11) is met by such an $\varepsilon$. So define $\varepsilon={ }_{\text {def }} \frac{\delta}{M+1}\left(=\frac{1}{2} \cdot \frac{2 \delta}{M+1}\right)$. With this $\varepsilon$ Requirement 4.1 is met. It remains to determine $L$.

Requirement 4.2 (Largeness of $L$ ) $L>0$ is so large that

$$
\bigcup_{\nu=2}^{m} Q_{\nu} \subset Q_{1} \text {. }
$$

The existence of such an $L$ can be easily verified by a glance on Fig. 5. For a formal proof put

$$
\begin{aligned}
& A={ }_{\operatorname{def}} \operatorname{conv}\left(\left(e_{d-1}+e_{d}+\Delta^{d-1}\right) \cup\left(-e_{d-1}+e_{d}+\Delta^{d-1}\right)\right), \\
& B={ }_{\operatorname{def}} \operatorname{conv}\left(A \cup\left((m-1) e_{d+1}+A\right)\right), \quad \text { and } \\
& \delta_{1}={ }_{\operatorname{def}} \operatorname{dist}\left(A, \operatorname{relbd}\left(e_{d}+M \cdot \Delta^{d-1}\right)\right) .
\end{aligned}
$$

$A$ is a $(d-1)$-polytope, $B$ is a right $d$-prism of height $m-1$ on $A$ containing $\bigcup_{\nu=2}^{m} Q_{\nu}$, and $\delta_{1}>0$ since $A$ and $e_{d}+M \cdot \Delta^{d-1}$ are compact and disjoint $\left(A \subset \operatorname{relint}\left(e_{d}+M \Delta^{d-1}\right)\right.$, by (8)). If $\frac{L}{\operatorname{dist}\left(o, \operatorname{relbd}\left(M \cdot \Delta^{d-1}\right)\right)}>\frac{m-1}{\delta_{1}}$, then the segment 
Fig. 5 A projection of Example 4.2 on the $\left(e_{d-1}, e_{d}\right)$-plane for $m=5$

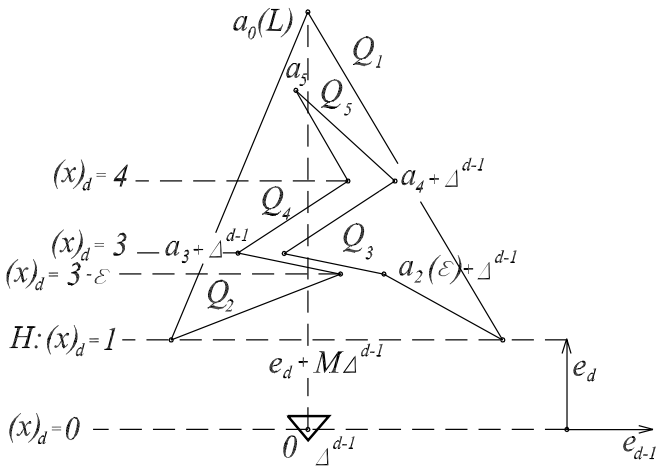

between $a_{0}(L)=(L+1) e_{d}$ and any point in $\operatorname{relbd}\left(M \cdot \Delta^{d-1}\right)$ does not meet $B$; hence for

$$
L>\frac{(m-1) \cdot \operatorname{dist}\left(o, \operatorname{relbd}\left(M \cdot \Delta^{d-1}\right)\right)}{\delta_{1}} .
$$

Requirement 4.2 is satisfied. So define

$$
L={ }_{\text {def }} \frac{(m-1) \cdot \operatorname{dist}\left(o, \operatorname{relbd}\left(M \cdot \Delta^{d-1}\right)\right)}{\delta_{1}}+1
$$

Having defined $Q_{1}, \ldots, Q_{m}$, put $Q={ }_{\text {def }} \operatorname{cl}\left(Q_{1} \backslash \bigcup_{\nu=2}^{m} Q_{v}\right)$. In view of (13), $Q$ is a zigzag hole drilled in $Q_{1}$ from its basis $e_{d}+M \cdot \Delta^{d-1}$ on (see Fig. 5), and the boundary of $Q$ is a polyhedral complex, henceforth denoted by $\mathcal{Q}$, combinatorially equivalent to the complex $\mathcal{P}$ of Example 4.1 above. Since bd $Q=|\mathcal{Q}|$ and int $Q=$ int $\mathcal{Q}$ (here int $\mathcal{Q}$ is in the sense of the polyhedral Jordan-Brouwer separation theorem (Theorem 1.1 above)), it follows that ext $\mathcal{Q}=\mathbb{R}^{d} \backslash(\mathcal{Q} \cup$ int $\mathcal{Q})$. (Here ext $\mathcal{Q}$ is in the sense of the polyhedral Jordan-Brouwer separation theorem (Theorem 1.1 above).)

The following implies that poldiam $(\operatorname{ext} \mathcal{Q})=m$ (compare Proposition 4.1 above $)$.

Proposition 4.2 It is possible to proceed via ext $\mathcal{Q}$ from any point $q_{0} \in \mathbb{R}^{d} \backslash$ $\left(H^{-} \cup Q_{1}\right) \subset \operatorname{ext} \mathcal{Q}$ to any point $q_{m} \in\left(\right.$ int $\left.B^{d}\left(a_{m}, 1\right)\right) \cap$ int $Q_{m} \subset \operatorname{ext} \mathcal{Q}$ (a 1-vicinity of $a_{m}$ in ext $\mathcal{Q}$ ) by a polygonal path of $m$ edges, but not by less than this.

Proof Let $d^{\prime} \in$ bd $Q_{1} \backslash\left\{a_{0}(L)\right\}$ satisfy $\left[q_{0}, d^{\prime}\right] \cap Q_{1}=\left\{d^{\prime}\right\}$. Such a point exists since $q_{0} \notin Q_{1}$. Since $q_{0} \notin H^{-}, d^{\prime} \notin e_{d}+M \cdot \Delta^{d-1}$, and $\left[q_{0}, d^{\prime \prime}\right] \cap Q_{1}=\left\{d^{\prime \prime}\right\}$ for all $d^{\prime \prime} \in$ cone $_{a_{0}(L)} d^{\prime}$. Let $d^{\prime \prime} \in\left(\right.$ cone $\left._{a_{0}(L)} d^{\prime}\right) \cap \hat{H}^{-}$; then (cone $\left.q_{0} d^{\prime \prime}\right) \cap$ int $\hat{H}^{-} \neq$ $\emptyset$ and $\left(\right.$ cone $\left._{q_{0}} d^{\prime \prime}\right) \cap Q_{1}=\emptyset$. Let $q_{1} \in\left(\right.$ cone $\left._{q_{0}} d^{\prime \prime}\right) \cap$ int $\hat{H}^{-}$, and define $\gamma={ }_{\text {def }}$ $\left\langle q_{0}, q_{1}, a_{2}(\varepsilon), a_{3}, a_{4}, \ldots, a_{m-1}, q_{m}\right\rangle$. Since $\left[q_{0}, q_{1}\right] \cap Q_{1}=\emptyset, \gamma$ is an $m$-gon from $q_{0}$ to $q_{m}$ via ext $\mathcal{Q}$. This proves the first part of the proposition.

To prove the second part observe that any polygonal path from $q_{0}$ to $q_{m}$ via $\operatorname{ext} \mathcal{Q}$ must reach a point $\hat{q}_{1} \in \hat{H}^{-}$before entering the "zigzag cave" $\bigcup_{\nu=2}^{m} Q_{\nu}$, which takes 
one edge at least. After this stage there remain to pass through the $m-1$ "gates" $a_{2}(\varepsilon)+\Delta^{d-1}, a_{3}+\Delta^{d-1}, \ldots, a_{m-1}+\Delta^{d-1}$ in this order (see Fig. 5 above) before reaching $q_{m}$, and this takes at least $m-1$ additional edges. The formal proof of this is analogous to the argument given for a similar assertion (Lemma 4.1) in the proof of Proposition 4.1 above, and we skip over the details with just one hint: Requirement 4.1 above (smallness of $\varepsilon>0$ ) ensures that it is impossible to reach from $\hat{q}_{1} \in \hat{H}^{-}$to the "gate" $a_{3}+\Delta^{d-1}$ via the "gate" $a_{2}(\varepsilon)+\Delta^{d-1}$ by just one edge (at least two edges are needed). It follows that a $k$-gon from $q_{0}$ to $q_{m}$ via ext $\mathcal{Q}$ has at least $1+(m-1)=m$ edges.

This example will be modified now to the remaining case $n \not \equiv 0(\bmod d)$ as follows. Assume first that $n \equiv 1(\bmod d)$, i.e., $n=m d+1$ and $\left\lceil\frac{n}{d}\right\rceil=m+1$. Take the (non-convex) body $Q$ described above and truncate its vertex $a_{0}(L)$ by a hyperplane $H^{\prime}$ parallel to $H$ (i.e., parallel to $\left.\operatorname{span}\left(e_{1}, \ldots, e_{d-1}\right)\right)$ passing close to $a_{0}(L)$; denote by $Q^{\prime}$ the resulting body. The boundary bd $Q^{\prime}$ of $Q^{\prime}$ is the body of a polyhedral $(d-1)$-complex, henceforth denoted by $\mathcal{Q}^{\prime}$, having $n=m d+1$ facets.

Claim 4.3 poldiam $\mathcal{Q}^{\prime}=m+1\left(=\left\lceil\frac{n}{d}\right\rceil\right)$.

Proof of Claim Denote by $F^{\prime}$ the new (horizontal) facet obtained by truncating $a_{0}(L)$ from $Q$ by $H^{\prime}$, let $q^{\prime}$ be a point in ext $\mathcal{Q}^{\prime}$ near the barycenter of $F^{\prime}\left(q_{0}^{\prime}\right.$ lies "above" $\left.F^{\prime}\right)$ and let $q \in$ (int $B^{d}\left(a_{m}, 1\right) \cap$ int $Q_{m}$ (a 1-vicinity of $a_{m}$ in ext $\left.\mathcal{Q}^{\prime}\right)$.

In order to reach from $q_{0}$ to $q_{m}$ by a polygonal path via ext $\mathcal{Q}^{\prime}$, it is necessary to reach first a point in $\hat{H}^{-}$in order to enter the zigzag cave $\bigcup_{\nu=2}^{m} Q_{\nu}$, and this can be done by not less than two edges, because $q_{0}$ lying near and above the barycenter of $F^{\prime}$ does not "see" directly any point of $\hat{H}^{-}$via ext $\mathcal{Q}^{\prime}$. After reaching a point in $\hat{H}^{-}$ there remain the $m-1$ "gates" $a_{2}(\varepsilon)+\Delta^{d-1}, a_{3}+\Delta^{d-1}, \ldots, a_{m-1}+\Delta^{d-1}$ to pass through in order to reach $q_{m}$, and this takes at least $m-1$ additional edges. Thus, in analogy with Proposition 4.2 above, it is possible to reach from $q_{0}$ to $q_{m}$ by a polygonal path of $2+m-1=m+1=\left\lceil\frac{n}{d}\right\rceil$ edges via ext $\mathcal{Q}^{\prime}$, but not by less than this. This proves Claim 4.3.

As to the remaining case $n \equiv l(\bmod d), 2 \leq l \leq m-1$, truncate any $l-1$ vertices of the facet $F^{\prime}$ of $Q^{\prime}$ by $l-1$ hyperplanes passing every one very close to the corresponding truncated vertex. The (non-convex) body $Q^{(l)}$ obtained has $n=m d+l$ facets and poldiam $\left(\operatorname{ext} Q^{(l)}\right)=m+1=\left\lceil\frac{n}{d}\right\rceil$.

This finishes Example 4.2.

\section{Two 2-PPMs in $\mathbb{R}^{3}$ Whose Interiors Cannot Be Tetrahedrized Without Additional Vertices}

As said in Remark 1.1, part (5) above, the interior of a simple closed polygon in the plane can be triangulated without additional vertices. The following two examples show that this property does not generalize to a $(d-1)-P P M$ in $\mathbb{R}^{d}$ for $d \geq 3$. 
Fig. 6 The six basic rectangular panels of Example 5.2

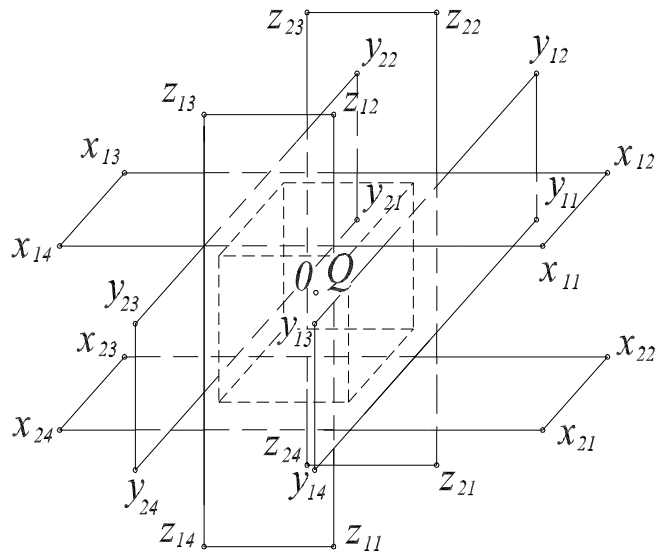

Example 5.1 The first example is the famous Schönhardt's twisted prism over a triangle, which is a simplicial 2-manifold $\mathcal{C}$ in $\mathbb{R}^{3}$ with six vertices combinatorially equivalent to the boundary complex of the 3-octahedron, first described in [28]. It is obtained from a 3-prism on a triangular basis by a (small), say clockwise, twist of one of the two bases. The three quadrilateral facets are thus broken at their three mutually disjoint diagonals and "bent in" so that the three dihedral angles at these three diagonals become concave (i.e., $>\pi$ ). The relative interiors of the other three diagonals are thus "pushed out" to lie entirely in ext $\mathcal{C}$. Clearly, any tetrahedron whose vertices are in vert $\mathcal{C}$ must have as an edge one of these three "outside diagonals". It follows that int $\mathcal{C} \cup \mathcal{C}$ cannot be tetrahedrized without additional vertices. This example was recently generalized in [27] to a twisted prism over any (convex) polygon, and much earlier it was generalized by [4] in a different, yet very simple direction. In [27], one can also find further discussion and references on this subject.

Let us present now another example which turns out to be combinatorially equivalent to the so called rhombicuboctahedron. Later on we shall see that, although Schönhardt's polyhedron is much simpler than our example below, this example is perhaps not devoid of interest because of another tetrahedrization-obstructing property it has over Schönhardt's and its generalizations described above.

Example 5.2 The idea is to put some mutually disjoint 2-dimensional rectangular panels $M_{1}, \ldots, M_{k}$ in $\mathbb{R}^{3}$ such that $o \in \mathbb{R}^{3} \backslash \bigcup_{i=1}^{k} M_{i}$ and every segment $[o, v]$, where $v$ is a vertex of some panel $M_{i}(1 \leq i \leq k)$, meets another panel $M_{j}(1 \leq j \leq k$, $j \neq i$ ), i.e., $o$ does not "see" any vertex of $\bigcup_{i=1}^{k} M_{i}$, and then to complete $\left\{M_{1}, \ldots, M_{k}\right\}$ to a $2-P P M \mathcal{C}$ by adding facets without additional vertices (i.e., $\left.\operatorname{vert} \mathcal{C}=\bigcup_{i=1}^{k} \operatorname{vert} M_{i}\right)$ so that $o \in \operatorname{int} \mathcal{C}$. The interior int $\mathcal{C}$ of such a 2-PPM cannot be tetrahedrized without additional vertices since a tetrahedron whose vertices are in vert $\mathcal{C}$ and whose edges do not meet any panel $M_{i}$ misses $o$. Here we take six congruent rectangular panels $X_{1}, X_{2}, Y_{1}, Y_{2}, Z_{1}, Z_{2}$ of size $1 \times 3$ in parallel to the coordinates planes, defined as follows (cf. Fig. 6).

Let $\varepsilon>0$ be small, define $x_{11}=\left(1 \frac{1}{2},-\frac{1}{2}, \frac{1}{2}+\varepsilon\right), x_{12}=\left(1 \frac{1}{2}, \frac{1}{2}, \frac{1}{2}+\varepsilon\right), x_{13}=$ $\left(-1 \frac{1}{2}, \frac{1}{2}, \frac{1}{2}+\varepsilon\right), x_{14}=\left(-1 \frac{1}{2},-\frac{1}{2}, \frac{1}{2}+\varepsilon\right)$, and put $X_{1}={ }_{\operatorname{def}} \operatorname{conv}\left\{x_{1 i}: 1 \leq i \leq 4\right\}$. 
$X_{1}$ is a rectangle of size $1 \times 3$ parallel to the $(x, y)$-plane at $z$-distance $\frac{1}{2}+\varepsilon$ from it, whose long (resp., short) edges run in parallel to the $x$-axis (resp., $y$-axis). Let $X_{2}$ be the rectangle whose vertices $x_{2 i}, 1 \leq i \leq 4$, are obtained from the vertices $x_{1 i}, 1 \leq i \leq 4$, merely by negation of the $z$-coordinate from $\frac{1}{2}+\varepsilon$ to $-\frac{1}{2}-\varepsilon$. $X_{2}$ is a translate of $X_{1}$ by $-1-2 \varepsilon$ in direction parallel to the $z$-axis, hence the $z$-distance between $X_{1}$ and $X_{2}$ is $1+2 \varepsilon$.

Let $Y_{1}$ (resp., $Y_{2}$ ) be the rectangle obtained from $X_{1}$ (resp., $X_{2}$ ) by the cyclic permutation of the coordinates $(x, y, z) \rightarrow(y, z, x)$ (a rotation of $120^{\circ}$ around the line aff $((0,0,0),(1,1,1)))$, and let $Z_{1}$ (resp., $\left.Z_{2}\right)$ be the rectangle obtained from $X_{1}$ (resp., $\left.X_{2}\right)$ by the cyclic permutation of the coordinates $(x, y, z) \rightarrow(z, x, y)$. We label the vertices of $Y_{1}$ (resp., $\left.Y_{2}\right)$ by $y_{1 i}$ (resp., $\left.y_{2 i}\right)(1 \leq i \leq 4)$ and those of $Z_{1}$ (resp., $Z_{2}$ ) by $z_{1 i}$ (resp., $\left.z_{2 i}\right)(1 \leq i \leq 4)$, as depicted in Fig. 6. The six rectangular panels obtained are mutually disjoint. Clearly, if $\varepsilon>0$ is small enough (say $\left.\varepsilon=\frac{1}{10}\right)$, then the origin is "hidden" from all their $24(=6 \times 4)$ vertices $x_{i j}, y_{i j}, z_{i j}(1 \leq i \leq 2,1 \leq j \leq 4)$, i.e., every segment between $o$ and one of these vertices meets some panel in its relative interior. The standard unit $(1 \times 1 \times 1)$ cube $Q$ of $\mathbb{R}^{3}$ centered at $o$ depicted in Fig. 6 by broken lines is situated between every pair of parallel panels, and each facet of $Q$ is at distance $\varepsilon$ apart from the closest panel. Note that $Y_{1} \cup Y_{2} \subset \operatorname{conv}\left(\operatorname{aff} X_{1} \cup\right.$ aff $\left.X_{2}\right), X_{1} \cup X_{2} \subset \operatorname{conv}\left(\operatorname{aff} Z_{1} \cup\right.$ aff $\left.Z_{2}\right)$, and $Z_{1} \cup Z_{2} \subset \operatorname{conv}\left(\operatorname{aff} Y_{1} \cup\right.$ aff $\left.Y_{2}\right)$. Let $M_{1}, M_{2} \in\left\{X_{1}, X_{2}, Y_{1}, Y_{2}, Z_{1}, Z_{2}\right\}$ be two non-parallel panels. To every short edge $m_{1}$ of $M_{1}$ corresponds the long edge $m_{2}$ of $M_{2}$ which is closest to it, and vice versa, $m_{1}$ is the short edge of $M_{1}$ which is closest to $m_{2}$. The lines aff $m_{1}$, aff $m_{2}$ are parallel, hence conv $\left(m_{1} \cup m_{2}\right)$ is an isosceles trapezoid whose bases are of lengths 1 and 3 . While the couple $\left(M_{1}, M_{2}\right)$ ranges over all couples of non-parallel panels and $\left(m_{1}, m_{2}\right)$ ranges over all couples of such (short-long) edges, we obtain $6 \cdot 2=12$ congruent (spatial) isosceles trapezoids, such as (cf. Fig. 6) $z_{13} z_{12} x_{11} x_{14}, x_{21} x_{22} y_{11} y_{14}, y_{11} y_{12} z_{21} z_{22}$, etc. Finally define eight triangles as follows. To each vertex $v$ of the central cube $Q$ correspond three of the panels $X_{i}, Y_{i}, Z_{i}(1 \leq i \leq 2)$ which are closest to it - each at distance $\varepsilon$ from $v$; on each of these three panels there is a vertex which is closest to $v$. The convex hull of these three vertices is a triangle $T_{v}$ that corresponds to $v$, e.g., $x_{11} z_{11} y_{13}$ is such a triangle (Fig. 6). The 26 panels thus defined (6 rectangular, 12 trapezoidal and 8 triangular) form the set of facets of a polyhedral 2-pseudomanifold $\mathcal{C}$ in $\mathbb{R}^{3}$ satisfying $f_{0}(\mathcal{C})=24, f_{1}(\mathcal{C})=6 \cdot 4+3 \cdot 8=48$, and $\left.f_{2}(\mathcal{C})=26\right)$. Note that $f_{0}(\mathcal{C})-f_{1}(\mathcal{C})+f_{2}(\mathcal{C})=2$, hence $\mathcal{C}$ is spherical. In fact, $\mathcal{C}$ is combinatorially equivalent to the face complex of the rhombicuboctahedron (an Archimedean solid) as one can easily check. Since $o \in \operatorname{int} \mathcal{C}$ and $o$ is "hidden" from every vertex of $\mathcal{C}$ (by some rectangular panel) int $\mathcal{C}$ cannot be tetrahedrized without additional vertices. End of Example 5.2.

The 2-PPMs $\mathcal{C}$ just described have the following tetrahedrization-obstructing property not shared by Schönhardt's 2-PPM and its generalizations:

Property A There is a point $p \in \operatorname{int} \mathcal{C}$ (in our case $p=o$ ) such that $p$ does not see any vertex of $\mathcal{C}$ via int $\mathcal{C}$. In other words, $p$ is "hidden" from every vertex of $\mathcal{C}$ by some facet of $\mathcal{C}$. 
Property A implies that every tetrahedron with vertices in vert $\mathcal{C}$ misses $p$ or intersects $\operatorname{ext} \mathcal{C}$, hence int $\mathcal{C} \cup \mathcal{C}$ cannot tetrahedrized without additional vertices. Schönhardt's 2-PPM (and its generalizations) show that this does not go vice versa: it cannot be tetrahedrized without additional vertices, and yet every point in its interior sees some vertex via the interior (as the reader may easily check). In view of Example 5.2 above, the following questions arise:

\section{Question 5.1}

(a) Is there a $2-P P M \mathcal{C}$ in $\mathbb{R}^{3}$ having Property A with \#(vert $\left.\mathcal{C}\right)<24$ ?

(b) If there is such a $2-P P M$, what is the minimal number of its vertices?

\section{The Role Played by Jordan's Separation Theorem for Simple Closed Polygons in the Proof of the Eulerian Relation $v-e+f=2$ for Planar Graphs}

As is well known, a connected planar graph $G=\langle V, E\rangle$ embedded in the plane with $v={ }_{\text {def }} \# V$ vertices and $e==_{\text {def }} \# E$ edges, whose complement $\mathbb{R}^{2} \backslash|E|$ has $f$ connectivity components ("domains"), satisfies the Eulerian relation

$$
v-e+f=2 \text {. }
$$

It seems to be much less known that a (rigorous) proof of (15) demands in an essential way the full statement of Jordan's Curve Theorem and its variant: a tree embedded in the plane does not separate it. In fact, of the many proofs of (15) encountered in the literature (notably textbooks in discrete mathematics, which are supposed to be rigorous), usually using induction on $e$, only a small fraction mentions Jordan's theorem at all, and even these mostly just pay lips service to the theorem, deliberately overlooking some delicate points. It is therefore desirable to prove (15) first for the relatively simple case where $G$ is embedded piecewise linearly in the plane, because in this case Jordan's theorem is particularly simple to prove, as mentioned in the introduction ("raindrop" proof). As far as we could check, no such treatment appears in the literature. The main stages for such a treatment may go as follows:

(a) Note first that, when $G$ is a simple closed polygon, (15) reduces to Jordan's theorem.

(b) Prove that a tree $T$ (piecewise linearly) embedded in the plane does not separate the plane. The proof is by induction on the numbers of edges of $T$. This proves (15) for the case that $G$ is a tree.

(c) Let $T$ be a spanning tree of $G$. Use (b) as an initial induction step (induction on $e$ ), and then "put back" the edges of $G$ not appearing in $T$ one by one.

(d) Use Jordan's theorem for simple closed polygons in the plane to prove that at each stage an edge is added $f$ increases (exactly) by 1, thus keeping (15) in balance.

We may come to this topic in a subsequent paper [26]. What is the natural generalization of (15) for polyhedral $(d-1)$-complexes (piecewise linearly embedded) in $\mathbb{R}^{d}$ ? 
Guided by the model given above for the proof of (15) it seems that the JordanBrouwer Separation Theorem for $(d-1)$-PPMs enunciated in Theorem 1.1 above will have some role in proving such a generalization.

\section{References}

1. Alexander, J.W.: On the subdivision of 3-space by a polyhedron. Proc. Nat. Acad. Sci. U.S.A. 10, 6-8 (1924)

2. Alexander, J.W.: An example of a simply connected surface bounding a region which is not simply connected. Proc. Nat. Acad. Sci. U.S.A. 10, 8-10 (1924)

3. Aleksandrov, P.S.: Combinatorial Topology (in three volumes). Translated from the Russian, 1947. Kombinatornaya Topologiya, by Harace Komm, Graylock Press, Rochester (1956). Reproduced by Dower Publications, New York (1998)

4. Bagemihl, F.: On indecomposable polyhedra. Am. Math. Mon. 55, 411-413 (1948)

5. Balinski, M.: On the graph structure of convex polyhedra in $n$-space. Pac. J. Math. 11, 431-434 (1961)

6. Barnette, D.W.: Coloring polyhedral manifolds. In: Goodman, J., Lutwak, E., Malkevitch, J., Pollack, R. (eds.) Discrete Geometry and Convexity, pp. 192-195. The New York Academy of Sciences, New York (1985)

7. Benson, R.V.: Euclidean Geometry and Convexity. McGraw-Hill, New York (1966)

8. Bertoglio, N., Chuaqui, R.: An elementary geometric nonstandard proof of the Jordan curve theorem. Geom. Dedic. 51, 15-27 (1994)

9. Boltyanski, V.G., Efremovic, V.A.: Anschauliche kombinatorische Topologie. Friedrich Vieweg \& Sohn, Braunschweig (1986)

10. Courant, R., Robbins, H.: What is Mathematics? 4th edn. Oxford University Press, London (1969)

11. Dostál, M., Tindell, R.: The Jordan curve theorem revisited. Jahresber. Dtsch. Math.-Ver. 80, 111-128 (1978)

12. Favard, J.: Espace et Dimension. Éditions Albin Michel, Paris (1950)

13. Grünbaum, B.: Convex Polytopes, 2nd edn. Springer, New York (2003)

14. Guggenheimer, H.: The Jordan curve theorem and an unpublished manuscript by Max Dehn. Arch. Hist. Exact Sci. 17, 193-200 (1977)

15. Hille, E.: Analytic Function Theory, vol. I. Ginn and Company, Boston (1959) (Chelsea, 1973)

16. Klee, V.: A $d$-pseudomanifold with $f_{0}$ vertices has at least $d f_{0}+(d-1)(d+2) d$-simplices. Houst. J. Math. 1, 81-86 (1975)

17. Kopperman, R., Meyer, P.R., Wilson, R.G.: A Jordan surface theorem for three-dimensional digital spaces. Discrete Comput. Geom. 6, 155-161 (1991)

18. Kupitz, Y.S., Martini, H.: Geometric graphs which are 1-skeletons of unstacked triangulated polygons. Discrete Math. 308, 5485-5498 (2008)

19. Kuratowski, K.: Introduction to Set Theory and Topology, 2nd edn. Pergamon Press and Polish Scientific Publications, Warsaw (1972)

20. Lawson, T.: Topology: A Geometric Approach. Oxford Graduate Texts in Mathematics, vol. 9. Oxford University Press, London (2003)

21. Menger, K.: Zur allgemeinen Kurventheorie. Fund. Math. 10, 95-115 (1927)

22. Menger, K.: Kurventheorie. Teubner, Leipzig (1932)

23. Moise, E.E.: Geometric Topology in Dimension 2 and 3. Springer, New York (1977)

24. Munkres, J.R.: Elements of Algebraic Topology. Addison-Wesley, Reading (1984)

25. Perles, M.A., Martini, H., Kupitz, Y.S.: On the polygonal diameter of the interior (exterior) of a simple closed polygon in the plane, in preparation

26. Perles, M.A., Martini, H., Kupitz, Y.S.: The role played by Jordan's theorem for simple closed polygons in the proof of the Eulerian relation $v-e+f=2$ in the plane, in preparation

27. Rambau, J.: On a generalization of Schönhardt's polyhedron. In: Combinatorial and Computational Geometry. MSRI Publications, vol. 52, pp. 501-516. Cambridge University Press, Cambridge (2005)

28. Schönhardt, E.: Über die Zerlegung von Dreieckspolyedern in Tetraeder. Math. Ann. 98, 309-312 (1928)

29. Schulte, E., Wills, J.M.: A polyhedral realization of Felix Klein's map $(3,7)_{8}$ on a Riemann surface of genus 3. J. Lond. Math. Soc. 32, 539-547 (1985) 
30. Skiena, S.: Implementing Discrete Mathematics, Combinatorics and Graph Theory with Mathematica. Addison-Wesley, Reading (1990)

31. Spanier, E.H.: Algebraic Topology. McGraw-Hill, New York (1966). (There is also a corrected reprint by Springer, Berlin, 1995)

32. Thomassen, C.: The Jordan-Schönflies theorem and the classification of surfaces. Am. Math. Mon. 99, 116-130 (1992)

33. Weisstein, E.: Menger's $n$-Arc Theorem. From MathWorld-A Wolfram Web Resource. http:// mathworld.wolfram.com/Mengersn-ArcTheorem.html

34. Whitney, H.: Congruent graphs and the connectivity of graphs. Am. J. Math. 54, 150-168 (1932) 